\title{
Continuous-Time Optimization Approach for Medium-Range Production Scheduling of a Multi-Product Batch Plant
}

\author{
Xiaoxia Lin and Christodoulos A. Floudas* $\quad$ Sweta Modi and Nikola M. Juhasz \\ Department of Chemical Engineering ATOFINA Chemicals, Inc. \\ Princeton University 900 First Avenue \\ Princeton, NJ 08544-5263 King of Prussia, PA 19406-0936
}

\begin{abstract}
The medium-range production scheduling problem of a multi-product batch plant is studied. The methodology consists of a decomposition of the whole scheduling period to successive short horizons. A mathematical model is proposed to determine each short horizon and the products to be included. Then a novel continuous-time formulation for short-term scheduling of batch processes with multiple intermediate due dates is applied to each time horizon selected, leading to a large-scale mixed-integer linear programming (MILP) problem. Special structures of the problem are further exploited to improve the computational performance. An integrated graphical user interface implementing the proposed optimization framework is presented. The effectiveness of the proposed approach is illustrated with a large-scale industrial case study that features the production of thirty five different products according to a basic 3-stage recipe and its variations by sharing ten pieces of equipment.
\end{abstract}

Keywords: medium-range scheduling, multi-product batch process, decomposition, continuoustime formulation, MILP.

\section{Introduction}

In multi-product batch plants, different products are manufactured via the same or similar sequence of operations by sharing available pieces of equipment, intermediate materials and other production

\footnotetext{
${ }^{*}$ Author to whom all correspondence should be addressed; Tel: (609) 258-4595; Fax: (609) 258-0211; E-mail: floudas@titan.princeton.edu.
} 
resources. They have long been accepted for the manufacture of chemicals that are produced in small quantities and for which the production process or the demand pattern is likely to change. The inherent operational flexibility of this type of plant provides the platform for great savings reflected in a good production schedule.

The research area of production scheduling and planning of multi-product and multi-purpose chemical processes has received great attention in the last decade. One of the most recent reviews of the related works is that of $\mathrm{Shah}^{1}$, which first examined different techniques for optimizing production schedules at individual sites, with an emphasis on formal mathematical methods, and then focused on progress in the overall planning of production and distribution in multi-site flexible manufacturing systems. In another review, Pekny and Reklaitis ${ }^{2}$ discussed the nature and characteristics of the scheduling/planning problems in chemical processing industries and pointed out the key implications for the solution methodology for these problems. Most of the work in this area has dealt with either the long-term planning problem or the short-term scheduling problem. Long-term planning or capacity expansion problems involve identifying the timing, location of additional facilities over a relatively long time horizon ${ }^{3}$. Short-term scheduling models address detailed sequencing of various operational tasks over short time periods. All of the mathematical models in the literature can be classified into two main groups based on the time representations. Early attempts rely on the discretization of the time horizon into a number of intervals of equal duration $^{4,5}$. This approach is a discrete approximation of the time horizon and results in an unnecessary increase of the overall size of the mathematical model. Recent work aims at developing efficient continuous-time models $6,7,8,9,10,11,12$. However, it should be pointed out that all slot-based formulations ${ }^{6,7,8}$ restrict the time representation and result by definition in suboptimal solutions. Floudas and coworkers ${ }^{13,14,15}$ proposed a novel true continuous-time mathematical model for the general short-term scheduling problem of batch, continuous and semicontinuous processes, which is the basis of the work presented in this paper. Lin and Floudas ${ }^{16}$ further extended this model to incorporate scheduling issues in the design and synthesis of multipurpose batch processes.

The rest of this paper is organized as follows. We will first present the problem investigated in 
this work. Then the overall framework is proposed and detailed formulations of a decomposition model and a short-term scheduling model are discussed. Computational results from an industrial case study are also given. At the end, an integrated graphical user interface implementing the proposed optimization framework is presented.

\section{Problem Description}

In this work, we investigate the medium-range production scheduling problem of a multi-product batch plant, which is defined as follows: Given (i) the production recipe (i.e., the processing times for each task at the suitable units, and the amount of the materials required for the production of each product), (ii) the available units and their capacity limits, (iii) the available storage capacity for each of the materials, and (iv) the medium-range time horizon under consideration, then the objective is to determine (i) the optimal sequence of tasks taking place in each unit, (ii) the amount of material being processed at each time in each unit, and (iii) the processing time of each task in each unit, so as to satisfy the market requirements expressed as specific amounts of products at given time instances within the time horizon.

In the batch plant investigated, there are three types of operations: Operations 1, 2 and 3. Up to sixty different products can be produced. For each of them, one of the processing recipes shown in Figure 1 is applied. The recipes are represented in the form of State-Task Network $(\mathrm{STN})^{4}$, in which the state node is denoted by a circle and the task node by a rectangle box. Some products share the same Operation 1 step.

The plant has three types of units: four Type 1 units (Units 1 4) for Operation 1, three Type 2 units (Units 5 7) for Operation 2, and three Type 3 units (Units 8 10) for Operation 3. The information on which units are suitable for each product is given. Type 1 units and Type 3 units are utilized in a batch mode, while Type 2 units operate in a continuous mode. The capacity limit of each Type 1 unit varies from one product to another, while the capacity limit of each Type 3 unit is the same for all suitable products. The processing time or processing rate of each task in 
the suitable units is also specified. When switched from one type of product to another, the units need cleaning up between. The data above are relatively fixed, which seldom change over a long period.

[Figure 1 about here.]

The time horizon considered for production scheduling is as long as a whole month. Customer orders are distributed throughout the time horizon with specified amounts, due dates and priorities. We assume (i) no limitation on raw materials, and (ii) unlimited storage capacity for all materials based on analysis of the specific situation in the plant.

\section{Overall Framework}

The overall methodology for solving the medium-range production scheduling problem is to decompose the large and complex problem to smaller short-term scheduling sub-problems in successive time horizons. The flowchart is shown in Figure 2. The first step is to input relevant data. Then, a mathematical model for the determination of the current time horizon and corresponding products that should be included is formulated and solved. According to the solution of the decomposition model, a short-term scheduling model is formulated using the information on customer orders, inventory levels and processing recipes. The resulting MILP problem is a large-scale complex problem which requires a large computational effort for its solution and exhibits difficulties in obtaining global optimality. It is solved iteratively by using cut-off values until a satisfactory feasible solution is obtained. Then the solution is output and the next time horizon is to be solved. The above procedure is applied iteratively until the whole scheduling period under consideration is finished.

[Figure 2 about here.] 


\section{Decomposition Model}

A key issue that arises in the rolling horizon approach described above is the determination of the time horizon and those products that should be considered for each short-term scheduling sub-problem. We develop a two-level mathematical formulation that effectively addresses this issue taking into account the trade-off between demands satisfaction, unit utilization and model complexity. In the first level, the time horizon is determined and the main products that should be considered for all processing steps are identified, while in the second level, additional products are identified to go through the Operation 1 step if needed.

\subsection{Level 1 Formulation}

The mathematical model involves the following notations:

Sets:

$D$ days;

$P$ products;

$R g$ Operation 1 groups;

$P_{r}$ products in Operation 1 group $(r)$ which share the same Operation 1 step;

$U d$ Type 3 units.

$U d_{p}$ Type 3 units suitable for product $(p)$.

\section{Parameters:}

nepd number of event points per day;

$d e m_{p, d}$ amount of demand for product $(p)$ due at day $(d)$; 
demt $_{p}$ total amount of demand for product $(p)$ under consideration;

suit $_{r}$ total number of Type 1 units suitable for production of Operation 1 group $(r)$;

suit $_{p}$ total number of other units suitable for production of product $(p)$;

$\operatorname{capd}_{u}$ capacity of Type 3 unit $(u)$;

$f t d_{p, u}$ fixed processing time in Type 3 unit $(u)$ for production of product $(p)$;

$\operatorname{prior}_{p, d}$ customer priority of demand for product $(p)$ due at day $(d)$;

$a h e a_{p, d}$ minimum number of days in advance to start processing to satisfy demand for product $(p)$ due on day $(d)$, which can be derived from the amount of the demand, unit capacities and task processing times;

$w t_{p}$ overall weight of product $(p)$ based on the priority, amount and due date of its first demand;

$c m p_{r}$ complexity index for processing of Operation 1 group $(r),>1$ for some products with special restrictions, $=1$ for others;

cplm maximum number of $w v(i, j, n)$ variables allowed as the complexity limit of the resulting scheduling model;

$\gamma_{d}$ upper bound on the ratio of the time for which the Type 3 units can be used for selected products over the time horizon;

$\alpha$ coefficient in objective function accounting for relative importance of products inclusion compared to horizon maximization.

\section{Variables:}


day $(d)$ binary, whether or not to include day $(d)$;

$\operatorname{prod}(p)$ binary, whether or not to include product $(p)$;

$r g(r)$ binary, whether or not Operation 1 group $(r)$ is included.

Based on the above definitions of parameters and variables, the following constraints and objective function are formulated:

$\underline{\text { Horizon Continuity }}$

$$
\operatorname{day}(d) \geq \operatorname{day}(d+1) \quad \forall d \in D, \quad d \neq d_{\text {last }} .
$$

These constraints ensure that successive days starting from the first one are selected to form a continuous time horizon.

$\underline{\text { Inclusion of products with demands in current horizon }}$

$$
\operatorname{prod}(p) \geq \operatorname{day}(d) \quad \forall p \in P, d \in D, \operatorname{dem}_{p, d}>0
$$

These constraints state that if there is a demand for product $(p)$ due on day $(d)$ and day $(d)$ is included in the current time horizon, then product $(p)$ should be considered for this horizon.

$\underline{\text { Inclusion of products with demands in the future }}$

$$
\operatorname{prod}(p) \geq \operatorname{day}\left(d-\text { ahea }_{p, d}\right) \quad \forall p \in P, d \in D, \text { ahea } a_{p, d}>0
$$

These constraints express the requirement that if a demand for product $(p)$ due on day $(d)$ needs $a h e a_{p, d}$ day(s) in advance to start processing in order to satisfy the due date and day $\left(d-a h e a_{p, d}\right)$ 
is included in the horizon, then product $(p)$ has to be taken into account for this horizon.

$$
\operatorname{prod}(p) \geq \operatorname{day}(d-1) \quad \forall p \in P, d \in D, \text { prior }_{p, d} \text { is high. }
$$

These constraints state that if a demand is of high priority, then it is pushed one day forward. These constraints are not necessary, however, this explicit consideration of demand priorities can lead to solutions that satisfy demands with high priorities to a larger extent.

Definition for variables $r g(r)$

$$
\begin{aligned}
& r g(r) \leq \sum_{p \in P_{r}} \operatorname{prod}(p) \quad \forall r \in R g \\
& r g(r) \geq \operatorname{prod}(p) \quad \forall r \in R g, p \in P_{r} .
\end{aligned}
$$

These constraints relate $r g(r)$ variables with corresponding $\operatorname{prod}(p)$ variables. An Operation 1 group $(r)$ is included, that is, $r g(r)=1$, if and only if one or more of the products that belong to this Operation 1 group are included, that is, $\operatorname{prod}(p)=1$.

$\underline{\text { Model complexity limit }}$

$$
\left[\sum_{r \in R g} r g(r) \cdot \text { suit }_{r} \cdot \mathrm{cmp}_{r}+\sum_{p \in P} \operatorname{prod}(p) \cdot \mathrm{suit}_{p}\right] \cdot \sum_{d \in D} d a y(d) \cdot \text { nepd } \leq \mathrm{cplm}
$$

The left hand side of this constraint gives an estimate of the number of $w v(i, j, n)$ binary variables (see scheduling model in next section) in the resulting scheduling problem. Because it can be used to represent the scale and complexity of the scheduling problem, this constraint keeps the scheduling problem under tractable size by imposing an upper bound on the total number of $(i, j, n)$ combinations. 
$\underline{\text { Type } 3 \text { unit utilization limit }}$

$$
\sum_{p \in P} \operatorname{prod}(p) \frac{d e m t_{p}}{\sum_{u \in U d_{p}} \operatorname{capd}_{u}} \min _{u \in U d_{p}}\left\{f t d_{p, u}\right\}\left|U d_{p}\right| \leq \gamma_{d} \sum_{d \in D} d a y(d) \cdot 24 \cdot|U d| .
$$

The left hand side represents a lower bound on the total number of hours for which the Type 3 units are utilized to satisfy demands of selected products under consideration, for example, within two weeks. Thus, this constraint limits the selected products by considering the utilization of Type 3 units.

Objective: Maximization of duration of horizon and products included

$$
\sum_{d \in D} d a y(d)+\alpha \sum_{p \in P} w t_{p} \cdot \operatorname{prod}(p) .
$$

The first term in the objective maximizes the duration of the time horizon, while the second term aims at including as many products as possible. $\alpha$ is used to balance the relative importance of these two targets.

The formulation described above is a mixed-integer nonlinear programming (MINLP) problem due to the bilinear terms of binary variables in constraint (7). We introduce additional binary variables $p d(p, d)$ and $r d(r, d)$ and the following constraints to replace the bilinear products of $\operatorname{prod}(p) \cdot \operatorname{day}(d)$ and $r g(r) \cdot \operatorname{day}(d)$.

Definition for variables $p d(p, d)$

$$
\begin{aligned}
& p d(p, d) \leq \operatorname{prod}(p) \quad \forall p \in P, d \in D \\
& p d(p, d) \leq \operatorname{day}(d) \quad \forall p \in P, d \in D \\
& p d(p, d) \geq \operatorname{prod}(p)+\operatorname{day}(d)-1 \quad \forall p \in P, d \in D .
\end{aligned}
$$


This set of linear constraints are equivalent to $p d(p, d)=\operatorname{prod}(p) \cdot d a y(d)$ because $p d(p, d)$ are binary variables.

$\underline{\text { Definition for variables } r d(r, d)}$

$$
\begin{aligned}
& r d(r, d) \leq r g(r) \quad \forall r \in R g, d \in D \\
& r d(r, d) \leq d a y(d) \quad \forall r \in R g, d \in D \\
& r d(r, d) \geq r g(r)+\operatorname{day}(d)-1 \quad \forall r \in R g, d \in D .
\end{aligned}
$$

Similarly, these constraints are equivalent to $r d(r, d)=r g(r) \cdot d a y(d)$.

Now, constraint (7) can be reformulated as follows:

$\underline{\text { Reformulation of constraint on model complexity limit }}$

$$
\left\{\sum_{r \in R g}\left[\text { suit }_{r} \cdot c m p_{r} \sum_{d \in D} r d(r, d)\right]+\sum_{p \in P}\left[\text { suit }_{p} \sum_{d \in D} p d(p, d)\right]\right\} \cdot \text { nepd } \leq c p l m .
$$

By including constraints (10)-(15) and replacing constraint (7) with constraint (16), the original MINLP problem is transformed to an MILP problem and can be solved to global optimality effectively.

\subsection{Level 2 Formulation}

After the time horizon and the main products are determined in the first level, a second level mathematical model is formulated to investigate the utilization of the Type 1 units, in which the first step in the processing sequence, the Operation 1 step, is performed, and to include additional products, if necessary, to go through the Operation 1 step to ensure that the Type 1 units are utilized efficiently.

The second-level mathematical model involves the following notations: 
Sets:

$R g$ Operation 1 groups;

$P_{r}$ products in Operation 1 group $(r)$;

Ur Type 1 units;

$U r_{r}$ Type 1 units suitable for Operation 1 group $(r)$.

Parameters:

$s l_{p}$ 0-1 parameter to indicate whether product $(p)$ is selected in the first level;

$d_{e m} t_{r}$ total amount of demand for post-Operation1 intermediate material of Operation 1 group $(r)$

$\operatorname{capr}_{r, u}$ capacity of Type 1 unit ( $u$ ) for processing of Operation 1 group $(r)$;

$f t r_{r, u}$ fixed processing time in Type 1 unit $(u)$ for processing of Operation 1 group $(r)$;

$\gamma_{r}$ lower bound on the fraction of the time horizon for which the Type 1 units are utilized;

$H$ duration of the time horizon determined in the first level.

\section{Variables:}

$r g(r)$ binary, whether or not to include Operation 1 group $(r)$.

The following constraints and objective function are formulated: 
$\underline{\text { Inclusion of products selected in first level }}$

$$
r g(r) \geq s l_{p} \quad \forall r \in R g, \quad p \in P_{r} .
$$

These constraints ensure that if a product is selected in the first level, that is, $s l_{p}=1$, then the Operation 1 group to which this product belongs is included, that is, $r g(r)=1$.

$\underline{\text { Type } 1 \text { unit utilization }}$

$$
\sum_{r \in R g} r g(r) \frac{d e m t_{r}}{\sum_{u \in U r_{r}} \operatorname{capr}_{r, u}} \min _{u \in U r_{r}}\left\{f t r_{r, u}\right\}\left|U r_{r}\right| \geq \gamma_{r} \cdot H \cdot|U r| .
$$

The left hand side represents a lower bound on the total number of hours for which the Type 1 units are utilized to satisfy demands of selected Operation 1 groups. Thus, by imposing a lower bound such as the one on the above right hand side, this constraint expresses the requirement that enough products should be included to utilize the Type 1 units efficiently.

Objective: Minimization of Operation 1 groups included

$$
\sum_{r \in R g} r g(r)
$$

The objective in the second level is to minimize the total number of products included to limit the size and complexity of the resulting scheduling problem as long as efficient utilization of the Type 1 units is ensured.

The second level mathematical model leads to an MILP problem and can be solved easily. 


\section{Short-Term Scheduling Formulation}

\subsection{Basic Formulation}

After each time sub-horizon and corresponding products to be included are determined with the decomposition model, a continuous-time formulation for short-term scheduling with multiple intermediate due dates is applied. This formulation is based on Floudas, Ierapetritou and Hené's works $^{13,14,15}$, featuring the novel concept of event points and formulation of special sequence constraints.

The formulation is presented in details as follows:

Sets:

$I$ tasks;

$I_{j}$ tasks which can be performed in unit $(j)$;

$I_{s}$ tasks which either produce or consume process state $(s)$;

$J$ units;

$J_{i}$ units which are suitable for performing task $(i)$;

$N$ event points within the time horizon;

$S$ material states $(s)$.

\section{Parameters:}

$V_{i j}^{m i n}$ denotes the minimal capacity allowed of the specific unit $(j)$ when performing $\operatorname{task}(i)$

$V_{i j}^{\max }$ denotes the maximal capacity allowed of the specific unit $(j)$ when performing $\operatorname{task}(i)$ 
$\operatorname{tav}_{j}$ time when unit $(j)$ starts being available;

$\operatorname{dend}_{s}$ market requirement for state $(s)$ at the end of time horizon;

$\rho_{s i}^{p}, \rho_{s i}^{c}$ proportion of state $(s)$ produced, consumed from task $(i)$, respectively;

$\alpha_{i j}$ constant term of processing time of task $(i)$ in unit $(j)$;

$\beta_{i j} \quad$ variable term of processing time of task $(i)$ in unit $(j)$ expressing the time required by the unit to process one unit of material performing task $(i)$;

H time horizon;

valm $_{s}$ relative value of state $(s)$ in the sequence of materials for the corresponding product;

valp $_{s}$ relative value of the corresponding product indicating its priority;

vald $_{s}$ relative value of the corresponding product indicating its importance to fulfill future demands;

$t c l_{i i^{\prime}}$ clean-up times of units when switched from task $\left(i^{\prime}\right)$ to task $(i)$

$\operatorname{dint}_{s n}$ demand for state $(s)$ at event point $(n)$, which is specified based on relative time at which the demand has to be fulfilled, the number of stages required to produce the final product, and the number of other tasks that may take place in the same unit;

$d u e_{s n}$ due time for demand for state $(s)$ at event point $(n)$;

pri $_{s n}$ priority of demand for state $(s)$ at event point $(n)$; 
$\gamma$ constant coefficient in objective function balancing meeting demands with intermediate due dates and overall production.

\section{Variables:}

$w v(i, j, n)$ binary variables that assign the beginning of task $(i)$ in unit $(j)$ at event point $(n)$;

$y v(j, n)$ binary variables that assign the utilization of unit $(j)$ at event point $(n)$;

$B(i, j, n)$ amount of material undertaking task $(i)$ in unit $(j)$ at event point $(n)$;

$S T I(s)$ initial amount of state $(s)$;

$S T(s, n)$ amount of state $(s)$ at event point $(n)$;

$S T F(s)$ amount of state $(s)$ at the end of the horizon;

$D(s, n)$ amount of state $(s)$ delivered at event point $(n)$;

$S L(s, n)$ slack variable for the amount of state $(s)$ not meeting the demand at event point $(n)$;

$T^{s}(i, j, n)$ time that task $(i)$ starts in unit $(j)$ at event point $(n)$;

$T^{f}(i, j, n)$ time that task $(i)$ finishes in unit $(j)$ while it starts at event point $(n)$;

$\underline{\text { Allocation Constraints }}$

$$
\sum_{i \in I_{j}} w v(i, j, n)=y v(j, n) \quad \forall j \in J, n \in N
$$

These constraints express that in each unit $(j)$ and at an event point $(n)$ only one of the tasks that can be performed in this unit (i.e., $i \in I_{j}$ ) should take place. If unit $(j)$ is utilized at event point 
$(n)$, that is, $y v(j, n)$ equal 1 , then one of the $w v(i, j, n)$ variables should be activated. If unit $(j)$ is not utilized at event point $(n)$, then all $w v(i, j, n)$ variables take zero values, that is no assignments of tasks are made.

\section{$\underline{\text { Material Balances }}$}

$$
\begin{array}{r}
S T\left(s, n_{1 s t}\right)=S T I(s)+\sum_{i \in I_{s}} \rho_{s i}^{c} \sum_{j \in J_{i}} B\left(i, j, n_{1 s t}\right) \quad \forall s \in S \\
S T(s, n)=S T(s, n-1)-D(s, n)+\sum_{i \in I_{s}} \rho_{s i}^{p} \sum_{j \in J_{i}} B(i, j, n-1)+\sum_{i \in I_{s}} \rho_{s i}^{c} \sum_{j \in J_{i}} B(i, j, n) \\
\forall s \in S, n \in N \\
S T F(s)=S T\left(s, n_{\text {last }}\right)+\sum_{i \in I_{s}} \rho_{s i}^{p} \sum_{j \in J_{i}} B\left(i, j, n_{\text {last }}\right) \quad \forall s \in S
\end{array}
$$

where $\rho_{s i}^{c} \leq 0, \rho_{s i}^{p} \geq 0$ represent the proportion of state $(s)$ consumed by or produced from task (i), respectively. According to these constraints the amount of material of state $(s)$ at event point $(n)$ is equal to that at event point $(n-1)$ adjusted by any amounts delivered at event point $(n)$ and produced or consumed between the event points $(n-1)$ and $(n)$.

\section{$\underline{\text { Capacity Constraints }}$}

$$
\begin{gathered}
B(i, j, n) \geq V_{i j}^{m i n} w v(i, j, n) \quad \forall i \in I, \quad j \in J_{i}, \quad n \in N \\
B(i, j, n) \leq V_{i j}^{\max } w v(i, j, n) \quad \forall i \in I, \quad j \in J_{i}, \quad n \in N .
\end{gathered}
$$

These constraints express the minimal and maximal allowed capacity of a unit $(j)$, respectively, when performing task $(i)$. If $w v(i, j, n)$ equals one, then constraints (24) and (25) correspond to lower and upper bounds on the batch-size, $B(i, j, n)$. If $w v(i, j, n)$ equals zero, then $B(i, j, n)$ becomes zero.

In the multiproduct plant that we study, there is no physical restriction on the minimal capacity

of units and parameters $V_{i, j}^{m i n}$ are set to zero. However, if it is not allowed or not suitable to 
operate with small batch-sizes due to other considerations not included in this model, appropriate artificial values can be incorporated. There are also cases in which some units, for example, the Type 1 units, are always operated in full capacities to produce as much as possible. Then the two inequality constraints (24) and (25) are combined and give the following equality constraint:

$$
B(i, j, n)=V_{i j}^{\max } w v(i, j, n) \quad \forall i \in I_{r}, \quad j \in J_{i}, \quad n \in N
$$

where $I_{r}$ is the set of Operation 1 tasks.

\section{Duration Constraints}

$$
T^{f}(i, j, n)=T^{s}(i, j, n)+\alpha_{i j} w v(i, j, n)+\beta_{i j} B(i, j, n) \quad \forall i \in I, \quad j \in J_{i}, \quad n \in N
$$

where $\alpha_{i j}$ are the fixed processing times for batch tasks (Operation 1 and Operation 3) and zero for continuous tasks (Operation 2), $\beta_{i j}$ are the inverse of processing rates for continuous tasks and zero for batch tasks respectively. The duration constraints express the dependence of the time duration of task $(i)$ in unit $(j)$ at event point $(n)$ on the amount of material being processed. If $w v(i, j, n)$ equals one, then the last two terms in constraints (27) are added to $T^{s}(i, j, n)$. If $w v(i, j, n)$ equals zero, then the last two terms become zero due to the capacity constraints (24), (25) and hence $T^{f}(i, j, n)=T^{s}(i, j, n)$.

$\underline{\text { Sequence Constraints: }}$

$\underline{\text { Same task in the same unit }}$

$$
T^{s}(i, j, n+1) \geq T^{f}(i, j, n) \quad \forall i \in I, \quad j \in J_{i}, \quad n \in N, n \neq n_{\text {last }} .
$$

The sequence constraints (28) state that task $(i)$ starting in unit $(j)$ at event point $(n+1)$ should 
start after the end of the same task performed in the same unit which has already started at event point (n).

\section{Different tasks in the same unit}

The following set of constraints (29) establishes the relationship between the starting time of a task $(i)$ at event point $(n+1)$ and the ending time of task $\left(i^{\prime}\right)$ at event point $(n)$ when these tasks take place in the same unit $(j)$.

$$
\begin{array}{r}
T^{s}(i, j, n+1) \geq T^{f}\left(i^{\prime}, j, n\right)+t c l_{i i^{\prime}} w v(i, j, n)-H\left(1-w v\left(i^{\prime}, j, n\right)\right) \\
\forall j \in J, i \in I_{j}, \quad i^{\prime} \in I_{j}, \quad i \neq i^{\prime}, \quad n \in N, n \neq n_{\text {last }} .
\end{array}
$$

Constraints (29) are written for tasks $\left(i, i^{\prime}\right)$ that are performed in the same unit $(j)$. If both tasks are performed in the same unit they should be at most consecutive. This is expressed by constraints (29) because if $w v\left(i^{\prime}, j, n\right)=1$ which means that task $\left(i^{\prime}\right)$ takes place in unit $(j)$ at event point $(n)$, then the last term of constraint (29) becomes zero forcing the starting time of task $(i)$ in unit $(j)$ at event point $(n+1)$ to be greater than the ending time of task $\left(i^{\prime}\right)$ in unit $(j)$ at event point $(n)$ plus the required clean-up time; otherwise the right hand side of constraint (29) becomes negative and the constraint is trivially satisfied.

$\underline{\text { Different tasks in different units }}$

$$
\begin{aligned}
& T^{s}(i, j, n+1) \geq T^{f}\left(i^{\prime}, j^{\prime}, n\right)-H\left(1-w v\left(i^{\prime}, j^{\prime}, n\right)\right) \\
& \forall j, j^{\prime} \in J, i \in I_{j}, i^{\prime} \in I_{j^{\prime}}, i \neq i^{\prime}, \quad n \in N, n \neq n_{\text {last }} .
\end{aligned}
$$

Constraints (30) are written for different tasks $\left(i, i^{\prime}\right)$ that are performed in different units $\left(j, j^{\prime}\right)$ but take place consecutively according to the production recipe. Note that if task $\left(i^{\prime}\right)$ takes place in unit $\left(j^{\prime}\right)$ at event point $(n)$ (i.e., $\left.w v\left(i^{\prime}, j^{\prime}, n\right)=1\right)$, then we have $T^{s}(i, j, n+1) \geq T^{f}\left(i^{\prime}, j^{\prime}, n\right)$ and hence task $(i)$ in unit $(j)$ has to start after the end of task $\left(i^{\prime}\right)$ in unit $\left(j^{\prime}\right)$. Otherwise the right hand side becomes negative and the constraint is trivially satisfied. 
Constraints for Demands with Intermediate Due Dates

$$
D(s, n)+S L(s, n)=\operatorname{dint}_{s n} \quad \forall s \in S, \quad n \in N
$$

These constraints represent that products are delivered at event points when demands exist. The slack variables, $S L(s, n)$, are introduced to give more flexibility to the model in handling partial fulfillment of demands. Under feasible conditions, some or all of these variables can be fixed to zero to ensure that some or all of the demands within the time horizon are met.

\section{$\underline{\text { Due Dates Constraints }}$}

$$
T^{s}(i, j, n) \leq d u e_{s n} \quad \forall s \in S, i \in I_{s}, j \in J_{i}
$$

These constraints ensure the satisfaction of product demand by the corresponding due date.

\section{Constraints for Demands at the End of the Time Horizon}

$$
S T F(s) \geq \operatorname{dend}_{s} \quad \forall s \in S, \quad n \in N
$$

These constraints ensure the satisfaction of demands which should be met at the end of the time horizon.

$\underline{\text { Unit Available Time Constraints }}$

$$
T^{s}(i, j, n) \geq \operatorname{tav}_{j}-H(1-w v(i, j, n)) \quad \forall i \in I, j \in J_{i}, n \in N
$$

These constraints represent the requirement of not starting any task until the unit is available. When 
$w v(i, j, n)$ equals zero, which means the task is not activated, the constraint is relaxed and becomes trivial.

$\underline{\text { Time Horizon Constraints }}$

$$
\begin{aligned}
& T^{f}(i, j, n) \leq H \quad \forall i \in I, j \in J_{i}, n \in N \\
& T^{s}(i, j, n) \leq H \quad \forall i \in I, j \in J_{i}, n \in N .
\end{aligned}
$$

The time horizon constraints represent the requirement that every task start and end within the time horizon $(\mathrm{H})$.

Objective: Maximization of production

$$
-\sum_{s} \sum_{n} \operatorname{pri}_{s n} \cdot S L(s, n)+\gamma \sum_{s} \text { vald }_{s} \cdot \operatorname{valp}_{s} \cdot \operatorname{valm}_{s} \cdot S T F(s)
$$

The objective shown in (37) is the maximization of production in terms of relative value of all states minus the penalty term for not meeting demands at intermediate due dates.

\subsection{Additional Constraints}

The mathematical formulation described above results in an MILP problem, which can be solved by a commercial MILP solver such as CPLEX. Mainly because of its original concept of event points, the proposed formulation outperforms some other existing discrete-time and pseudo-continuoustime formulations in terms of reducing significantly the size of resulting mathematical programming problem and thus the required computational resources. However, solving the resulting MILP problems is still very challenging (e.g., the solutions require considerable CPU time for proof of global optimality), which reflects the inherent complexity of the specific physical conditions. To improve the modeling and solution, the following constraints are incorporated: 
$\underline{\text { Tighter Sequence Constraints for Operation } 1}$

Because Operation 1 is the first step in the task sequence for all products and we want to maximize the overall production in principle, the timing of the Operation 1 tasks can be enforced to be as tight as possible.

$\underline{\text { Same task in the same unit }}$

$$
\begin{array}{r}
T^{s}(i, j, n+1) \leq T^{f}(i, j, n)+H(2-w v(i, j, n)-w v(i, j, n+1)) \\
\forall i \in I_{r}, \quad j \in J_{i}, \quad n \in N, n \neq n_{\text {last }} .
\end{array}
$$

These additional sequence constraints for the same Operation 1 task in the same Type 1 unit, combined with constraints (28), enforce "zero-wait" condition on the task taking place at two consecutive event points. Namely, if $w v(i, j, n)=w v(i, j, n+1)=1$, that is, task (i) takes place in unit $(j)$ at both event point $(n)$ and $(n+1)$, then $T^{s}(i, j, n+1)=T^{f}(i, j, n)$, which state that in unit $(j)$, task $(i)$ starting at event point $(n+1)$ starts immediately after the end of the same task which has already started at event point (n); otherwise these constraints are relaxed.

Different tasks in the same unit

$$
\begin{array}{r}
T^{s}(i, j, n+1) \leq T^{f}\left(i^{\prime}, j, n\right)+t c l_{i i^{\prime}}+H\left(2-w v\left(i^{\prime}, j, n\right)-w v(i, j, n+1)\right) \\
\forall j \in J_{r}, i \in I_{j}, \quad i^{\prime} \in I_{j}, \quad i \neq i^{\prime}, \quad n \in N, n \neq n_{\text {last }}
\end{array}
$$

where $J_{r}$ is the set of Type 1 units.

According to these new constraints and constraints (29), if $w v\left(i^{\prime}, j, n\right)=w v(i, j, n+1)=1$ (that is, task $\left(i^{\prime}\right)$ takes place in Type 1 unit $(j)$ at event point $(n)$ and task (i) takes place in the same Type 1 unit at event point $(n+1))$, then $T^{s}(i, j, n+1)=T^{f}\left(i^{\prime}, j, n\right)+t c l_{i i^{\prime}}$ requiring that task $(i)$ in Type 1 unit $(j)$ at event point $(n+1)$ start immediately after task $\left(i^{\prime}\right)$ in the same Type 1 unit at event point $(n)$ ends and necessary cleaning is done for the Type 1 unit. Otherwise, these 
constraints are trivial.

\section{Restrictions on Binary Variables}

For each product, based on the information of overall amount of demands and maximal batchsizes of related tasks performed in suitable units, lower bounds on the total number of activated tasks can be specified for Operation 1, Operation 2 and Operation 3, respectively.

$$
\sum_{j \in J_{i}} \sum_{n \in N} w v(i, j, n) \geq m_{i} \quad \forall i \in I
$$

where $m_{i}$ are parameters calculated based on relevant data.

These constraints reduce the combinatorial complexity of the MILP problems and improve the computational performance.

\section{Special Restrictions}

\section{$\underline{\text { Restriction } 1}$}

It is given that Operation 1 for a specific product should run in one of the Type 1 units in campaign mode, namely, a prespecified minimum number, $c$, of batches needs to be performed consecutively once started. This arrangement is due to the comparatively long clean-up time for the Type 1 unit required to switch from this product to others. The corresponding constraints can be formulated as follows:

$$
\begin{array}{r}
w v(i, j, n) \geq w v(i, j, n-1) \quad \forall i \in I_{r E}, j \in J_{r E}, n \in N, 2 \leq n \leq c \\
w v(i, j, n) \geq w v(i, j, n-1)-\frac{1}{c-1} \sum_{n-c \leq n^{\prime} \leq n-2} w v\left(i, j, n^{\prime}\right) \\
\forall i \in I_{r E}, j \in J_{r E}, n \in N, n>c \\
w v(i, j, n) \leq w v(i, j, n-1) \quad \forall i \in I_{r E}, j \in J_{r E}, n \in N, n \geq n_{\text {last }}-c+2
\end{array}
$$

where $I_{r E}$ is the set of Operation 1 tasks and $J_{r E}$ is the set of Type 1 units dedicated to them. 
Constraints (41) and (42) state that if $w v(i, j, n-1)$ equals one and $w v(i, j, n-2) \sim w v(i, j, n-$ $c$ ), which do not all exist for $n$ less than $c$, are not all equal to one, which means task $(i)$ has taken place in unit $(j)$ at event point $(n-1)$ and there have been less than $c$ batches performed, then $w v(i, j, n)$ equals one, that is, task $(i)$ should take place in unit $(j)$ at event point $(n)$; otherwise, these constraints become trivial. Constraint (43) ensures that the Operation 1 task does not get started after event point $\left(n_{\text {last }}-c+1\right)$ because the Type 1 unit won't be able to perform $c$ batches in the remaining period.

\section{$\underline{\text { Restriction } 2}$}

A subset of the products are described as black. They are also required to be put together for Operation 2 and Operation 3 so as to avoid clean-up as much as possible.

$$
\begin{array}{r}
w v(i, j, n) \leq 2-\sum_{i^{\prime} \in I_{e d B} \cap I_{j}}\left[w v\left(i^{\prime}, j, n^{\prime}\right)+w v\left(i^{\prime}, j, n^{\prime \prime}\right)\right] \\
\forall i \in I_{e d N B}, j \in J_{i}, n, n^{\prime}, n^{\prime \prime} \in N, n^{\prime}<n<n^{\prime \prime}
\end{array}
$$

where $I_{e d B}$ and $I_{e d N B}$ are the set of Operation 2 and Operation 3 tasks for black products and non-black products, respectively. If Type 2 unit or Type 3 unit $(j)$ is utilized for black products at both event point $\left(n^{\prime}\right)$ and $\left(n^{\prime \prime}\right)$, then the right hand side of constraints (44) is zero, which states that no task for non-black products is allowed in the same unit at any event point $(n)$ between $\left(n^{\prime}\right)$ and $\left(n^{\prime \prime}\right)$. Otherwise, these constraints become trivial.

\section{Restriction 3}

Production of a special category of products only needs to go through Operation 1 and Operation 3. It is required that these Operation 1 and Operation 3 tasks run for 1-2 weeks in a campaign mode. Therefore, demands for this category of products within a period of time (e.g., one month) are put together. A Type 1 unit and a Type 3 unit are then dedicated to the "combined" Operation 1 task and Operation 3 task, respectively. The relative order of original tasks for different products

can be simply based on the due dates of demands. Production of this category of products is treated separately. The dedicated Type 1 unit and Type 3 unit are excluded from available resources for 
other products during the dedicated time intervals. This approach will be illustrated through the computational study in next section.

\section{Computational Study}

\subsection{Problem Overview}

The proposed rolling horizon approach is applied to an industrial case study in which detailed production schedules are to be determined to satisfy customer orders for various products distributed within a whole month.

The distribution of demands throughout the whole month under consideration is plot in Figure 3. There are five main categories of products and thirty five different products are required to be produced in this month. It is assumed that no final product is available at the beginning of the month. However, lower bounds on the amounts of initially available intermediate materials are provided.

[Figure 3 about here.]

The processing recipes to make these products are shown in Figure 1. The Operation 1 and Operation 3 steps are performed in a batch mode, while the Operation 2 step in a continuous mode. The processing time or processing rate of each step is dependent on both the product and the unit, with Operation 1, Operation 2, and Operation 3 in the ranges of 6-11 hours, 0.15-0.25 units/hour, and 12-16 hours, respectively. Capacities of the four Type 1 units vary from 1.125 units/batch to 3.5 units/batch, while capacities of the three Type 3 units are either 4.5 units/batch or 3.5 units/batch. The clean-up time required ranges from 2 hours to 36 hours, depending on the unit and the product sequence involved.

\subsection{Detailed Schedules}

"Campaign mode" production 
We first consider scheduling of the special category of products that require operation in the "campaign mode" (Category 4 in Figure 3). Demands for all products in this category in the whole month are grouped together. One Type 1 unit and one Type 3 unit are dedicated to the production of these products and the detailed schedule of the Operation 1 tasks and Operation 3 tasks for different products are determined based on their relative due dates. The starting time of production of all these products is determined so that all the due dates of the demands for these products can be satisfied. Then, the units and time intervals which are used are excluded from available resources for the production of other products. The relative schedule obtained for production of this category of products is shown in Figure 4.

[Figure 4 about here.]

The rolling horizon approach is then applied for the production of the remaining products to break down the large scheduling problem into several short-term scheduling sub-problems in successive time horizons. There are mainly two types of connections between consecutive time horizons: initial available time of units and intermediate materials. The decomposition and shortterm scheduling models are implemented with MINOPT ${ }^{17}$ and solved with CPLEX, a commercial MILP solver. The CPU time required to obtain each solution ranges from $15 \mathrm{~min}$ to about 7 hours on an HP J-2240 workstation.

\section{Horizon 1}

With parameter cplm of 1500 , the first horizon is determined to be the first 5 days of the month and 8 main products are identified to be included in this horizon according to Level 1 of the decomposition model. No additional product is identified to undergo the Operation 1 step from Level 2 of the decomposition model.

Twenty event points are used in the short-term scheduling model for this horizon, which leads to 1320 binary variables, 3968 continuous variables and 21912 constraints. Two feasible solutions are generated and the detailed schedule accepted is shown in Figure 5. 
[Figure 5 about here.]

\section{Horizon 2}

The second horizon is determined to be the next 5 days of the month, that is, from the 6th day to the 10th day, and 6 main products are identified to be included according to Level 1 of the decomposition model with $\mathrm{cplm}$ of 1000. No additional product is identified from Level 2 of the decomposition model.

Twenty three event points are used in the short-term scheduling model for this horizon, which leads to 897 binary variables, 2576 continuous variables and 14260 constraints. One feasible solution is obtained and accepted. The detailed schedule is shown in Figure 6. Note that the starting times of the Type 1 units correspond to the finishing times of the same units in the previous horizon.

[Figure 6 about here.]

\section{Horizon 3}

The third horizon is determined to be from the 11th day to the 14th day of the month and 10 main products are identified to be included according to Level 1 of the decomposition model with cplm of 1500. No additional product is identified from Level 2 of the decomposition model.

Nineteen event points are used in the short-term scheduling model for this horizon, which leads to 1216 binary variables, 3918 continuous variables and 22086 constraints. Three feasible solutions are obtained before the last one is accepted. The detailed schedule is shown in Figure 7.

[Figure 7 about here.]

\section{Horizon 4}

The fourth horizon is determined to be from the 15th day to the 19th day of the month and 8 main products are identified to be included according to Level 1 of the decomposition model with cplm of 2000. Seven additional product are identified to undergo the Operation 1 step from Level 2 of the decomposition model with $\gamma_{r}$ of $80 \%$. 
Twenty one event points are used in the short-term scheduling model for this horizon, which leads to 1827 binary variables, 5831 continuous variables and 37298 constraints. Three feasible solutions are obtained before the last one is accepted. The detailed schedule is shown in Figure 8.

[Figure 8 about here.]

\section{Horizon 5}

The fifth horizon is determined to be from the 20th day to the 24th day of the month and 9 main products are identified to be included according to Level 1 of the decomposition model with $\mathrm{cplm}$ of 2000. Five additional product are identified from Level 2 of the decomposition model with $\gamma_{r}$ of $30 \%$.

Twenty one event points are used in the short-term scheduling model for this horizon, which leads to 2016 binary variables, 6471 continuous variables and 41866 constraints. One feasible solution is obtained and accepted. The detailed schedule is shown in Figure 9.

[Figure 9 about here.]

\section{Horizon 6}

There are only six days remaining in the month. It is found that the demands in this remaining period can be fulfilled very easily and only 4.5 days is actually needed. Therefore, the last horizon is chosen to be 4.5 days starting from the 25 th day of the month and all of the 11 products for the remaining demands are included.

Ten event points are used in the short-term scheduling model for this horizon, which leads to 580 binary variables, 1820 continuous variables and 6723 constraints. Five feasible solutions are obtained before we accept the last one as a satisfactory schedule. The detailed schedule is shown in Figure 10.

[Figure 10 about here.] 


\subsection{Summary of Results}

As described in detail in the previous section, the decomposition model and the scheduling model are used iteratively, moving forward the scheduling horizon. In summary, the whole scheduling period is decomposed into six time horizons, each varying from 4 to 5 days long and including 6 to 15 products, as shown in Table 1.

[Table 1 about here.]

A sketch of the schedules obtained for the whole month is given in Figure 11. It should be noted that the Type 1 units are mostly idle towards the end of the whole period because no demands are specified for the coming period. The production schedules obtained for 28.5 days not only satisfy all demands in all categories, though some of the due dates are relaxed, but also produce $9.1 \%$ more than the demands in overall (see Table 2).

[Figure 11 about here.]

[Table 2 about here.]

Another important criterion for judging the production schedule is the efficiency of unit utilization. As shown in Table 3, the schedules obtained in this work ensure that the units, especially the Type 3 units, are utilized efficiently. The Type 3 units are utilized intensively throughout the whole period, which indicates that they are bottlenecks of the overall production as far as the demand structure in this case study is concerned.

[Table 3 about here.]

\section{Integrated Graphical User Interface}

A graphical user interface has been developed to integrate various components required to apply the proposed optimization framework to the medium-range production scheduling problem systematically and effectively. The flowchart is shown in Figure 12. After the user inputs all relevant 
data, which is stored in a database, the scheduling for products that require production in the "campaign mode" over a relatively long period is performed first. Then, based on the information in the database, the two-level decomposition mode is generated and then solved with an MILP solver, CPLEX. Next, the short-term scheduling model is formulated. The resulting MILP problem is solved iteratively by using cut-off values until a satisfactory feasible solution is obtained. Then the solution is output in readable formats, for example, the Gantt Chart, and the database is updated according to the solution to move towards the next time horizon. The above procedure is applied iteratively until the whole scheduling period is finished. The software is developed in extensive Visual Basic with M.S. Access supporting the database. It consists of the following five main functional modules.

[Figure 12 about here.]

\subsection{Data Manipulation}

All of the information is stored in a background database, and can be entered, changed or deleted through the user interface. Figure 13 shows the main window of the user interface with forms opened to access various data.

[Figure 13 about here.]

The important data include:

- Scheduling period;

- Orders, as shown in Figure 14, the amount, due date and priority for each customer order;

[Figure 14 about here.]

- Inventories of both final products and intermediate materials;

- Processing recipes, as shown in Figure 15, the processing steps required for making each product and other related information need to be specified; 
[Figure 15 about here.]

- Information of the units, as shown in Figure 16;

[Figure 16 about here.]

- Unit-product suitabilities, for example, Figure 17 shows the Type 1 Unit Suitability Form;

[Figure 17 about here.]

- Processing times/rates, for example, Figure 18 shows the Operation 3 Processing Time Form;

[Figure 18 about here.]

- Sequence-dependent clean-up requirements, for example, Figure 19 shows the transition table for the Type 1 units.

[Figure 19 about here.]

\subsection{Campaign-Mode Production Scheduling}

Products that are required to go through the campaign mode are handled separately. The appropriate products and suitable units are identified and a certain period of time is dedicated to the campaign-mode production of these products. Figure 20 shows schedule tables for the campaignmode production generated in the user interface.

[Figure 20 about here.] 


\subsection{Decomposition}

Based on the information in the database, the decomposition model is generated and then solved with an MILP solver, to determine the current time horizon and to identify products to be included for the scheduling problem. Figure 21 shows CPLEX solving the decomposition model and the results are presented in Figure 22.

[Figure 21 about here.]

[Figure 22 about here.]

\subsection{Short-term Scheduling}

According to the solution of the decomposition model, the short-term scheduling model is formulated to determine the detailed schedule for the current horizon. The resulting large-scale complex MILP problem is solved iteratively by using cut-off values until a satisfactory feasible solution is obtained. Figure 23 shows CPLEX solving the scheduling model.

[Figure 23 about here.]

\subsection{Results Output and Database Update}

The solution to the short-term scheduling model is organized in readable formats, including the schedule table and the Gantt Chart, and the database is updated accordingly. Figure 24 shows the Gantt chart representation of a schedule obtained in the user interface.

[Figure 24 about here.]

Furthermore, a wide variety of additional features are incorporated to make the graphical user interface as user-friendly as possible. For example, the user can request search functions on various data forms to access specific information efficiently. 


\section{Conclusions}

In this paper, the medium-range production scheduling problem of a multi-product batch plant is investigated. Three basic types of operation are involved and ten pieces of equipment are shared to produce up to sixty different products. The scheduling horizon considered is one month, even though longer horizons can be addressed with the proposed framework. The overall approach is to decompose the large and complex problem for the whole scheduling period into smaller shortterm scheduling sub-problems in successive time horizons. A two-level decomposition model is proposed to determine the current horizon and identify those products to be included. Then a continuous-time formulation for short-term scheduling of batch processes with multiple intermediate due dates is introduced. This procedure is applied iteratively until the whole scheduling period is completed. The effectiveness of this proposed rolling horizon approach is illustrated with computational results from an industrial case study. A graphical user interface developed to integrate various components to apply the proposed optimization framework systematically is also presented.

Acknowledgments: The authors gratefully acknowledge support from the National Science Foundation and from ATOFINA Chemicals, Inc.

\section{References}

(1) Shah, N. Single- And Multisite Planning and Scheduling: Current Status and Future Challenges. In Proceedings of the Third International Conference on Foundations of ComputerAided Process Operations, Snowbird, Utah, July 5-10, 1998. Pekny, J. F., Blau, G. E., Eds; CACHE-AIChE: New York, 1998; pp 75-90.

(2) Pekny, J. F.; Reklaitis, G. V. Towards the Convergence of Theory and Practice: A Technology Guide for Scheduling/Planning Methodology. In Proceedings of the Third International Con- 
ference on Foundations of Computer-Aided Process Operations, Snowbird, Utah, July 5-10, 1998. Pekny, J. F., Blau, G. E., Eds; CACHE-AIChE: New York, 1998; pp 91-111.

(3) Iyer, R. R.; Grossmann, I. E. A Bilevel Decomposition Algorithm for Long-Range Planning of Process Networks. Ind. Eng. Chem. Res. 1998, 37, 474.

(4) Kondili, E.; Pantelides, C. C.; Sargent, R. W. H. A General Algorithm for Short-Term Scheduling of Batch Operations - I. MILP Formulation. Comp. Chem. Engng. 1993, 17, 211.

(5) Shah, N.; Pantelides, C. C.; Sargent, R. W. H. A General Algorithm for Short-Term Scheduling of Batch Operations - II. Computational Issues. Comp. Chem. Engng. 1993, 17, 229.

(6) Pinto, J. M.; Grossmann, I. E. Optimal Cyclic Scheduling of Multistage Continuous Multiproduct Plants. Comp. Chem. Engng. 1994, 18, 797.

(7) Pinto, J. M.; Grossmann, I. E. A Continuous Time Mixed Integer Linear Programming Model for Short Term Scheduling of Multistage Batch Plants. Ind. Eng. Chem. Res. 1995, 34, 3037.

(8) Pinto, J. M.; Grossmann, I. E. A Logic-Based Approach to Scheduling Problems with Resource Constraints. Comp. Chem. Engng. 1997, 21, 801.

(9) Schilling, G.; Pantelides, C. C. A Simple Continuous-Time Process Scheduling Formulation and a Novel Solution Algorithm. Comp. Chem. Engng. 1996, 20, S1221.

(10) Zhang, X.; Sargent, R. W. H. The Optimal Operation of Mixed Production Facilities - General Formulation and Some Solution Approaches for the Solution. Comp. Chem. Engng. 1996, 20, 897.

(11) Mockus, L.; Reklaitis, G. V. Mathematical Programming Formulation for Scheduling of Batch Operations Based on Nonuniform Time Discretization. Comp. Chem. Engng. 1997, $21,1147$.

(12) Karimi, I. A.; McDonald, C. M. Planning and Scheduling of Parallel Semi-Continuous Processes. 2. Short-Term Scheduling. Ind. Eng. Chem. Res. 1997, 36, 2701. 
(13) Ierapetritou, M. G.; Floudas, C. A. Effective Continuous-Time Formulation for Short-Term Scheduling: 1. Multipurpose Batch Processes. Ind. Eng. Chem. Res. 1998a, 37, 4341.

(14) Ierapetritou, M. G.; Floudas, C. A. Effective Continuous-Time Formulation for Short-Term Scheduling: 2. Continuous and Semi-continuous Processes. Ind. Eng. Chem. Res. 1998b, 37, 4360.

(15) Ierapetritou, M. G.; Hené, T. S.; Floudas, C. A. Effective Continuous-Time Formulation for Short-Term Scheduling: 3. Multiple Intermediate Due Dates. Ind. Eng. Chem. Res. 1999, 38, 3446.

(16) Lin, X.; Floudas, C. A. Design, Synthesis and Scheduling of Multipurpose Batch Plants via an Effective Continuous-Time Formulation. Comp. Chem. Engng. 2001, 25, 665.

(17) Schweiger, C. A.; Floudas, C. A. MINOPT : A Modeling Language and Algorithmic Framework for Linear, Mixed-Integer, Nonlinear, Dynamic, and Mixed-Integer Nonlinear Optimization; Computer-Aided Systems Laboratory, Dept. of Chemical Engineering, Princeton University, NJ. 1997. 


\section{List of Figures}

1 State-Task Network of production recipes . . . . . . . . . . . . . . 36

2 Flowchart of the rolling horizon approach . . . . . . . . . . . 37

3 Distribution of demands . . . . . . . . . . . . . . . . . 38

4 Production schedule for a special category of products in campaign mode (U4, U10: units; Pc1-Pc6: products.) . . . . . . . . . . . . . . . . . . . . 39

5 Detailed schedule for Time Horizon 1 (U1-U10: units; P1-P8: products.) . . . . . . 40

6 Detailed schedule for Time Horizon 2 (U1-U10: units; P3-P13: products.) . . . . . 41

7 Detailed schedule for Time Horizon 3 (U1-U10: units; P3-P29: products.) . . . . . 42

8 Detailed schedule for Time Horizon 4 (U1-U10: units; P2-P29: products.) . . . . . 43

9 Detailed schedule for Time Horizon 5 (U1-U10: units; P2-P29: products.) . . . . . 44

10 Detailed schedule for Time Horizon 6 (U1-U10: units; P4-P28: products.) . . . . . 45

11 Sketch of production schedule for the whole month (U1-U10: units) . . . . . . . 46

12 Flowchart of the integrated user interface . . . . . . . . . . . . . . . 47

13 Data forms in the integrated graphical user interface . . . . . . . . . . . . . . . 48

14 Orders Form in the integrated graphical user interface . . . . . . . . . . . . . . . 49

15 Products Form in the integrated graphical user interface . . . . . . . . . . . . 50

16 Units Form in the integrated graphical user interface . . . . . . . . . . . . . . . 51

17 Type 1 Unit Suitability Form in the integrated graphical user interface . . . . . . . 52

18 Operation 3 Processing Time Form in the integrated graphical user interface . . . . 53

19 Type 1 Unit Transition Table in the integrated graphical user interface . . . . . . . 54

20 Schedule tables for campaign-mode production obtained in the integrated graphical user interface . . . . . . . . . . . . . . . . . . . . . 55

21 CPLEX solving the decomposition model in the integrated graphical user interface 56

22 Decomposition results shown in the integrated graphical user interface . . . . . . 57

23 CPLEX solving the short-term scheduling model in the integrated graphical user interface . . . . . . . . . . . . . . . . . . . 58

24 A schedule obtained in the integrated graphical user interface . . . . . . . . . . 59 


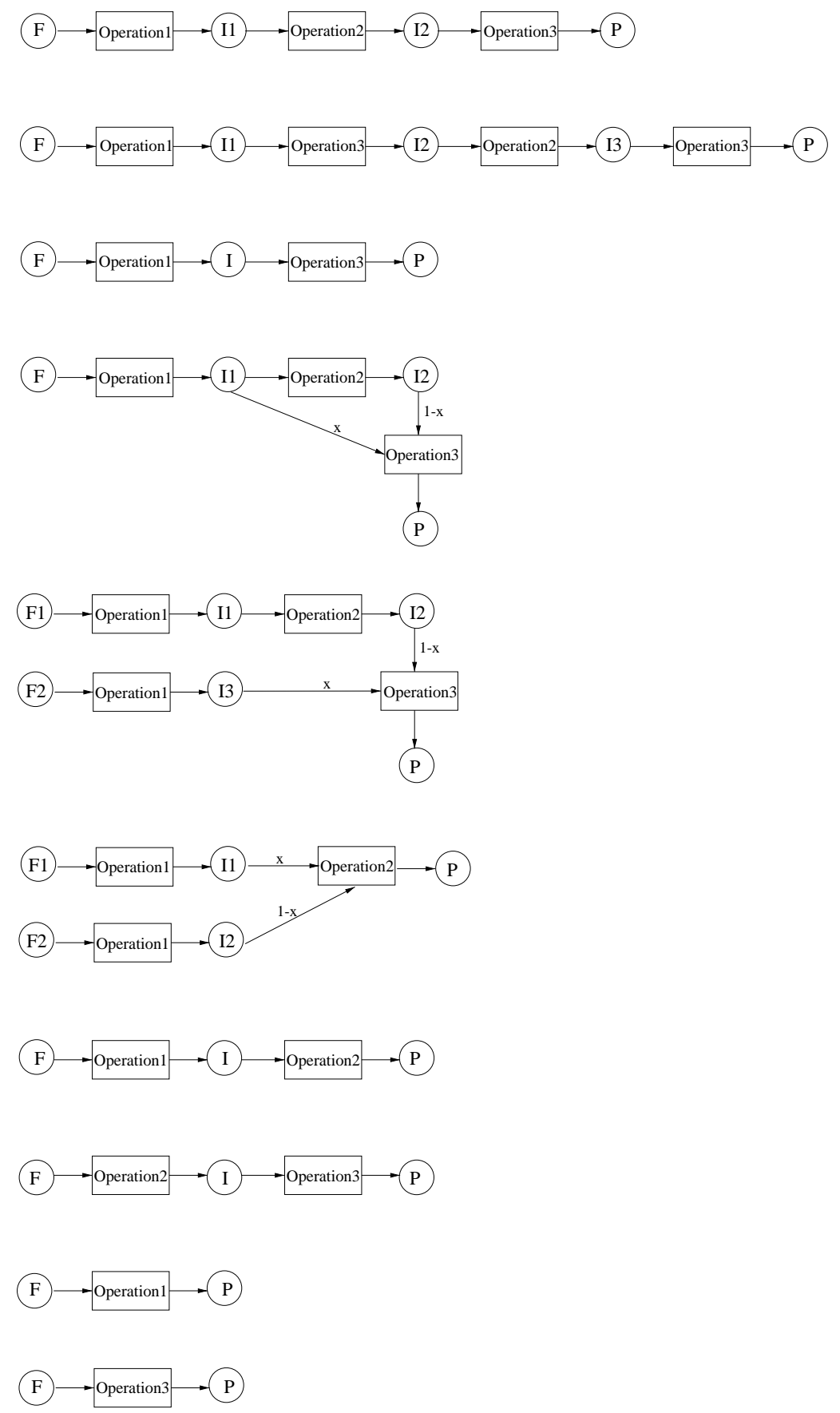

( a )

Figure 1: State-Task Network of production recipes 


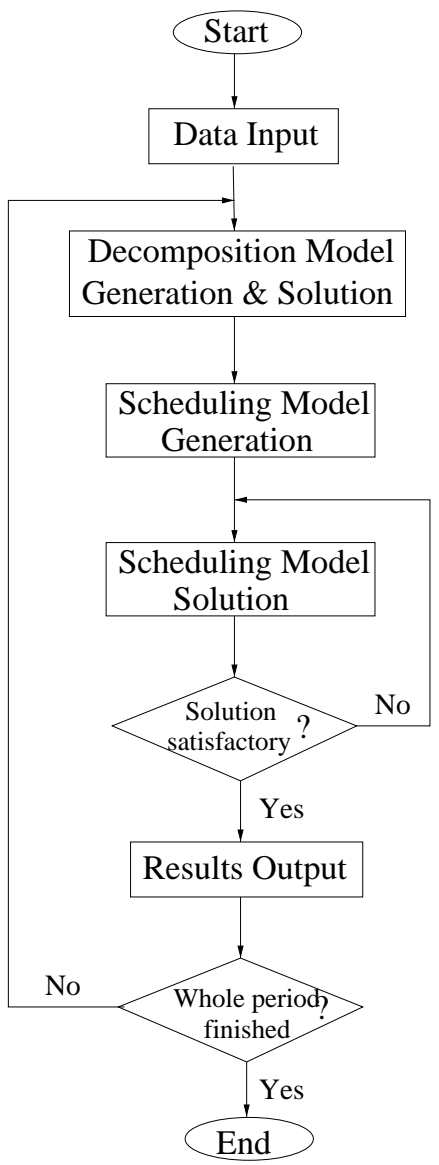

Figure 2: Flowchart of the rolling horizon approach 


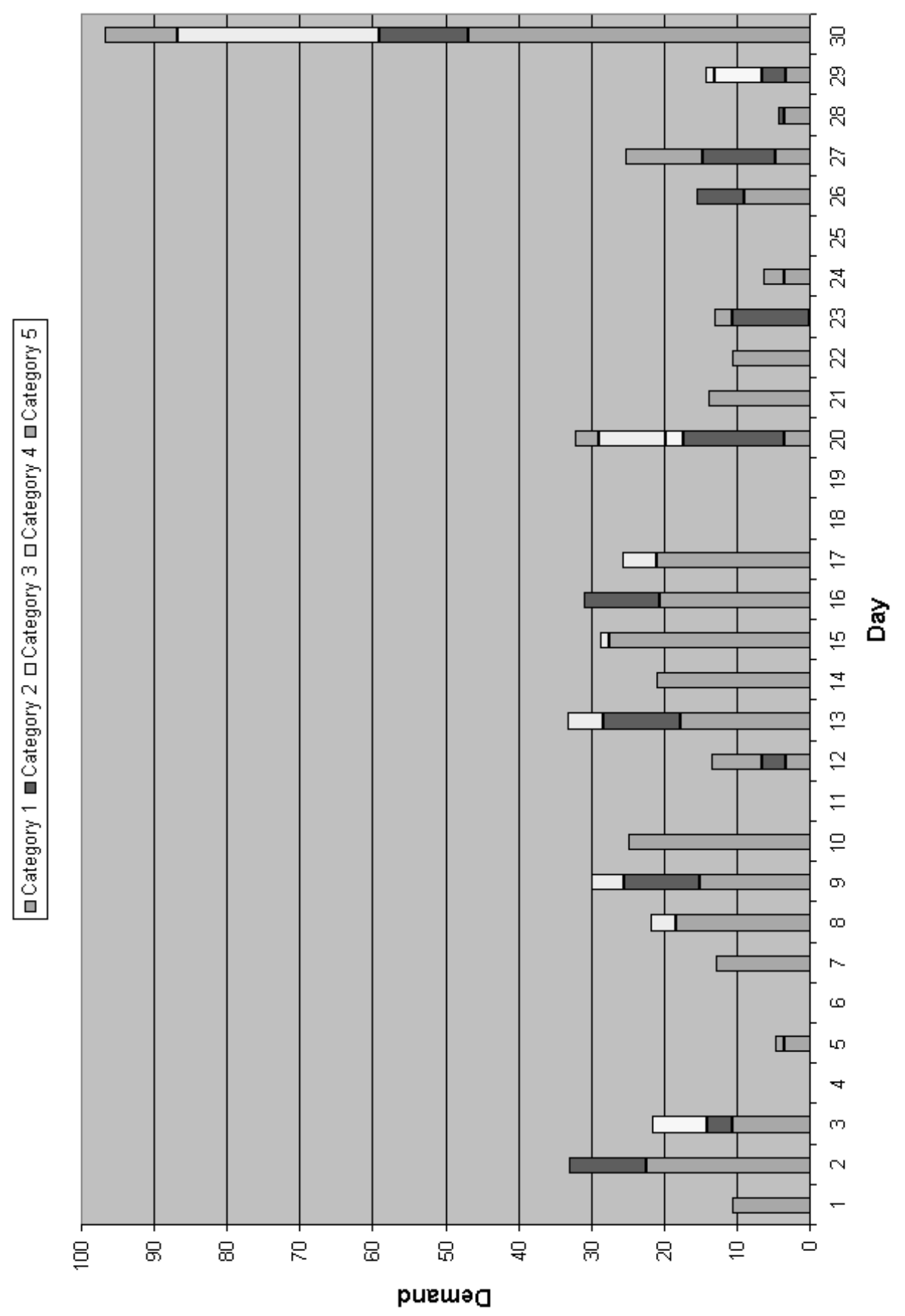

Figure 3: Distribution of demands 


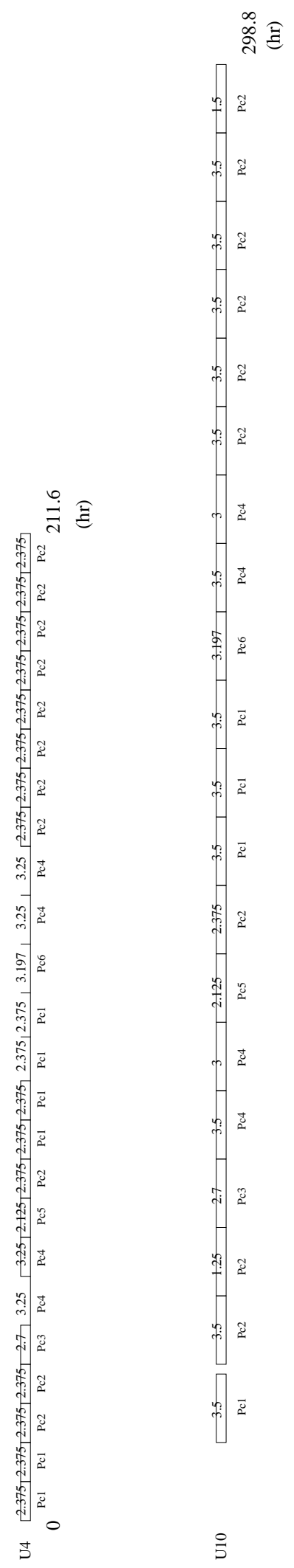

Figure 4: Production schedule for a special category of products in campaign mode (U4, U10: units; Pc1-Pc6: products.) 


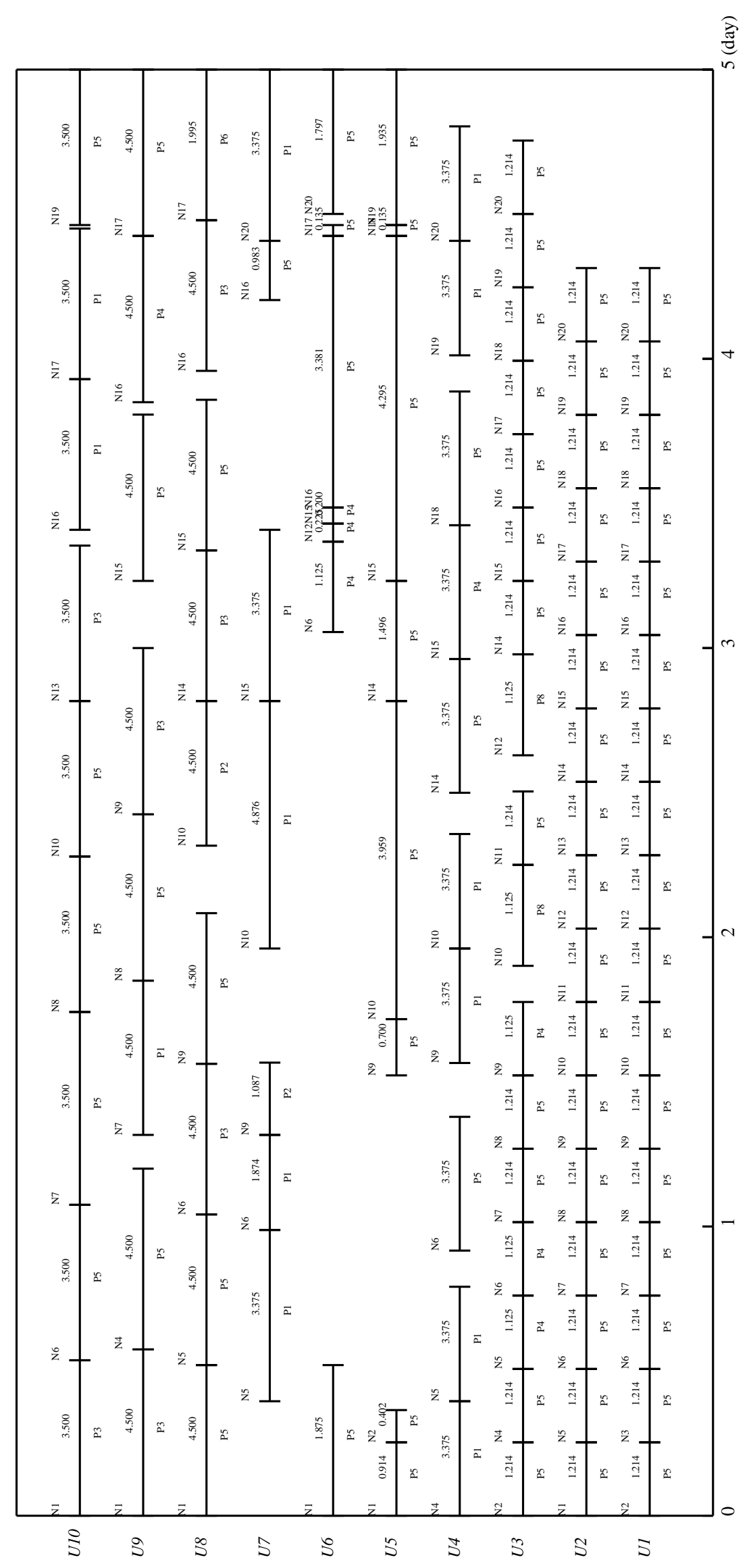

Figure 5: Detailed schedule for Time Horizon 1 (U1-U10: units; P1-P8: products.) 


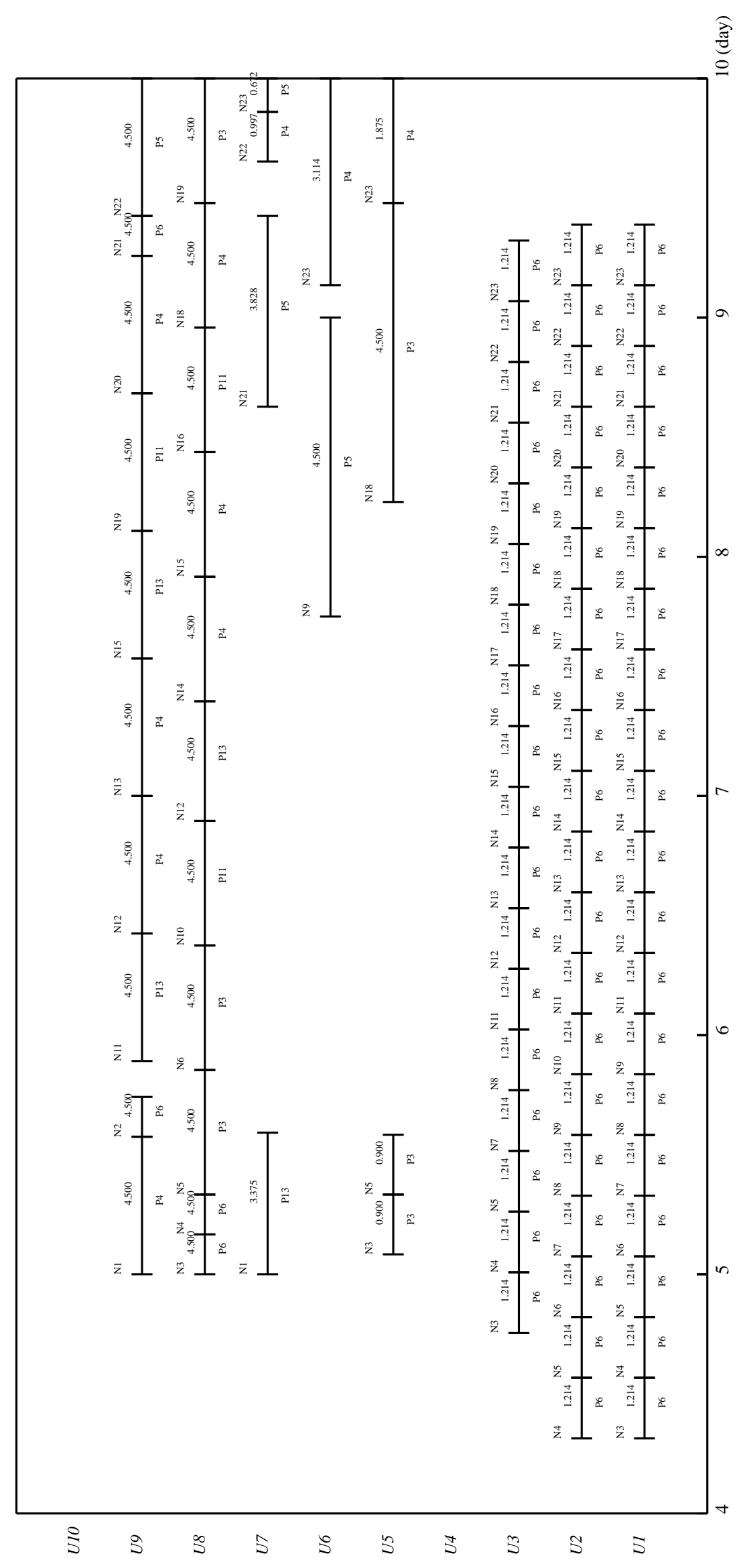

Figure 6: Detailed schedule for Time Horizon 2 (U1-U10: units; P3-P13: products.) 


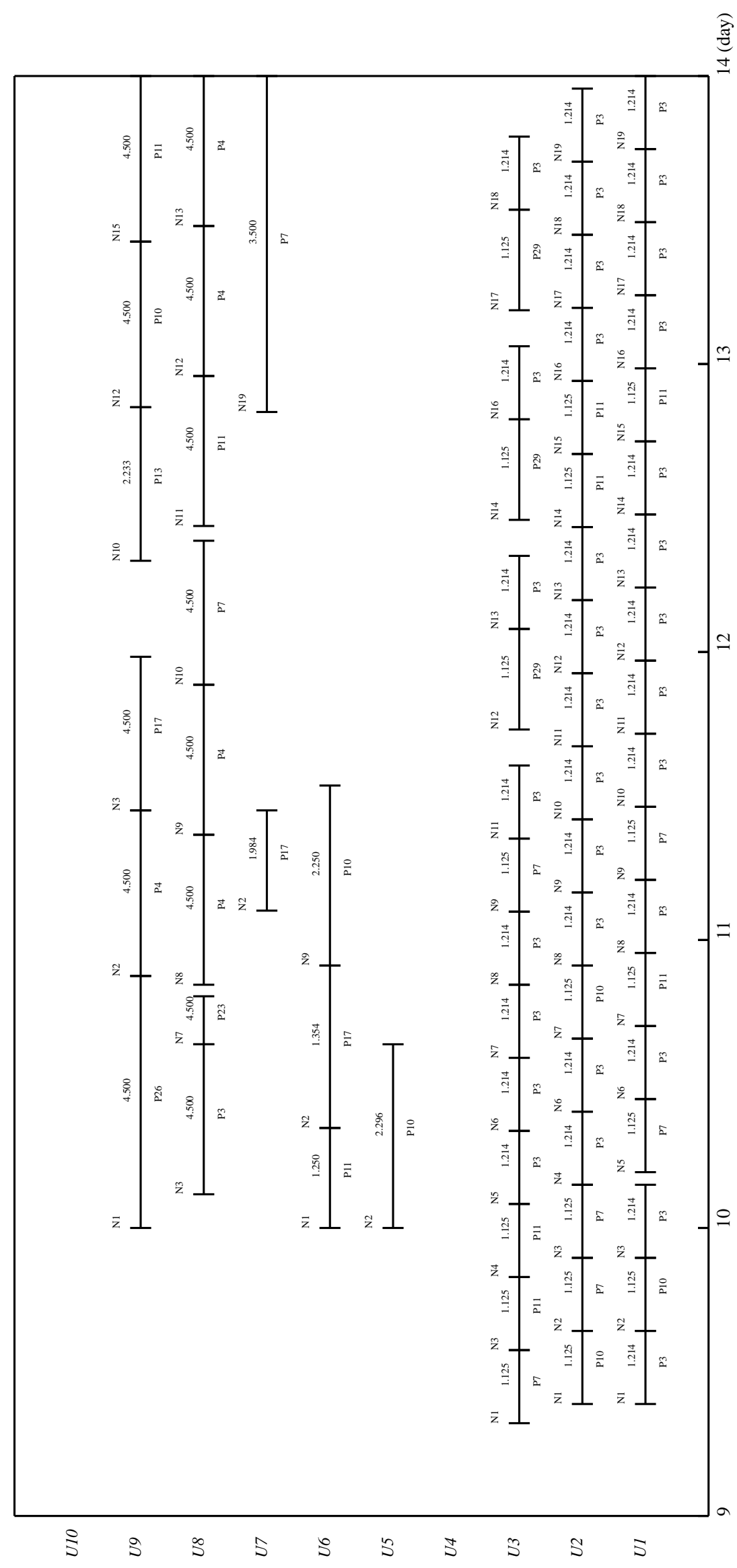

Figure 7: Detailed schedule for Time Horizon 3 (U1-U10: units; P3-P29: products.) 


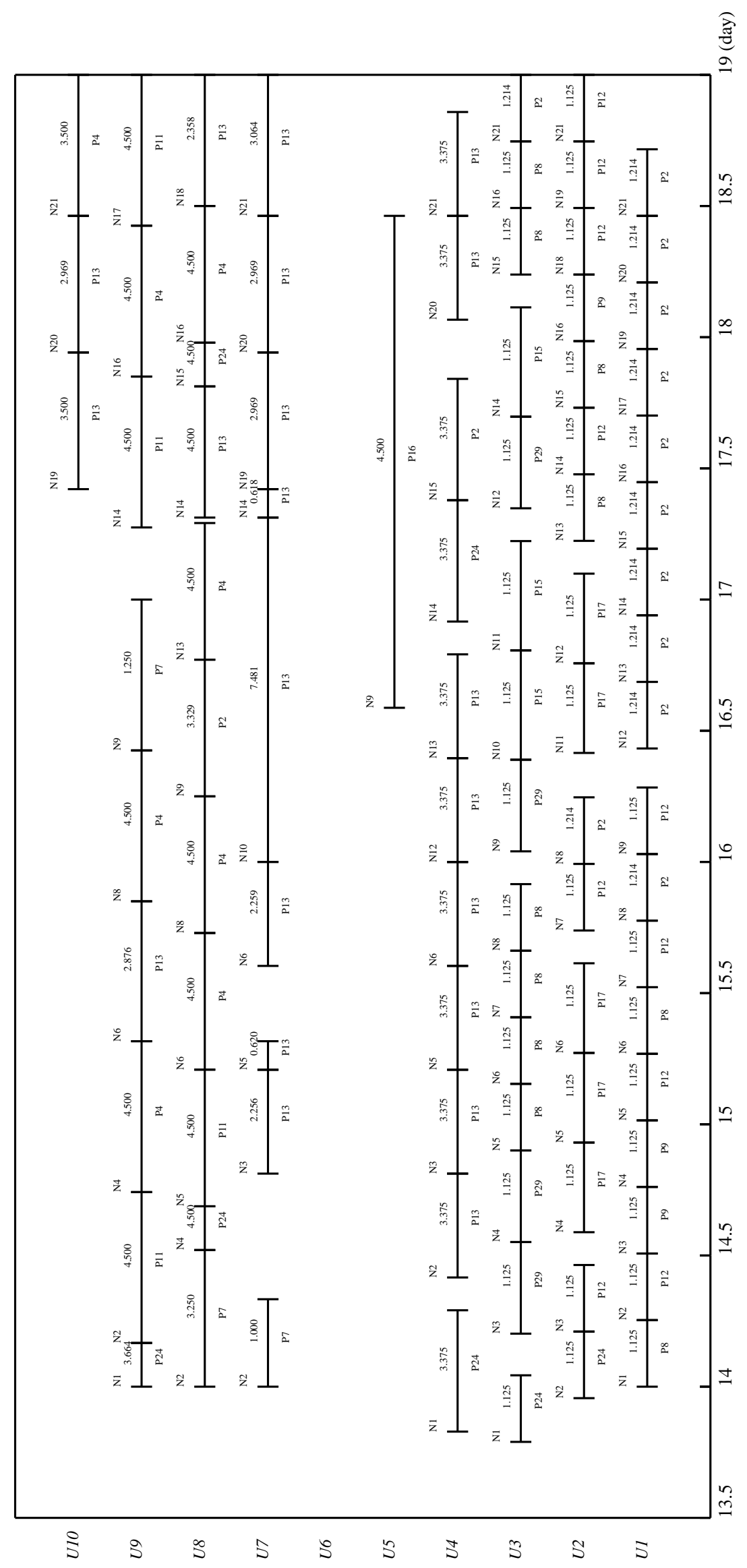

Figure 8: Detailed schedule for Time Horizon 4 (U1-U10: units; P2-P29: products.) 


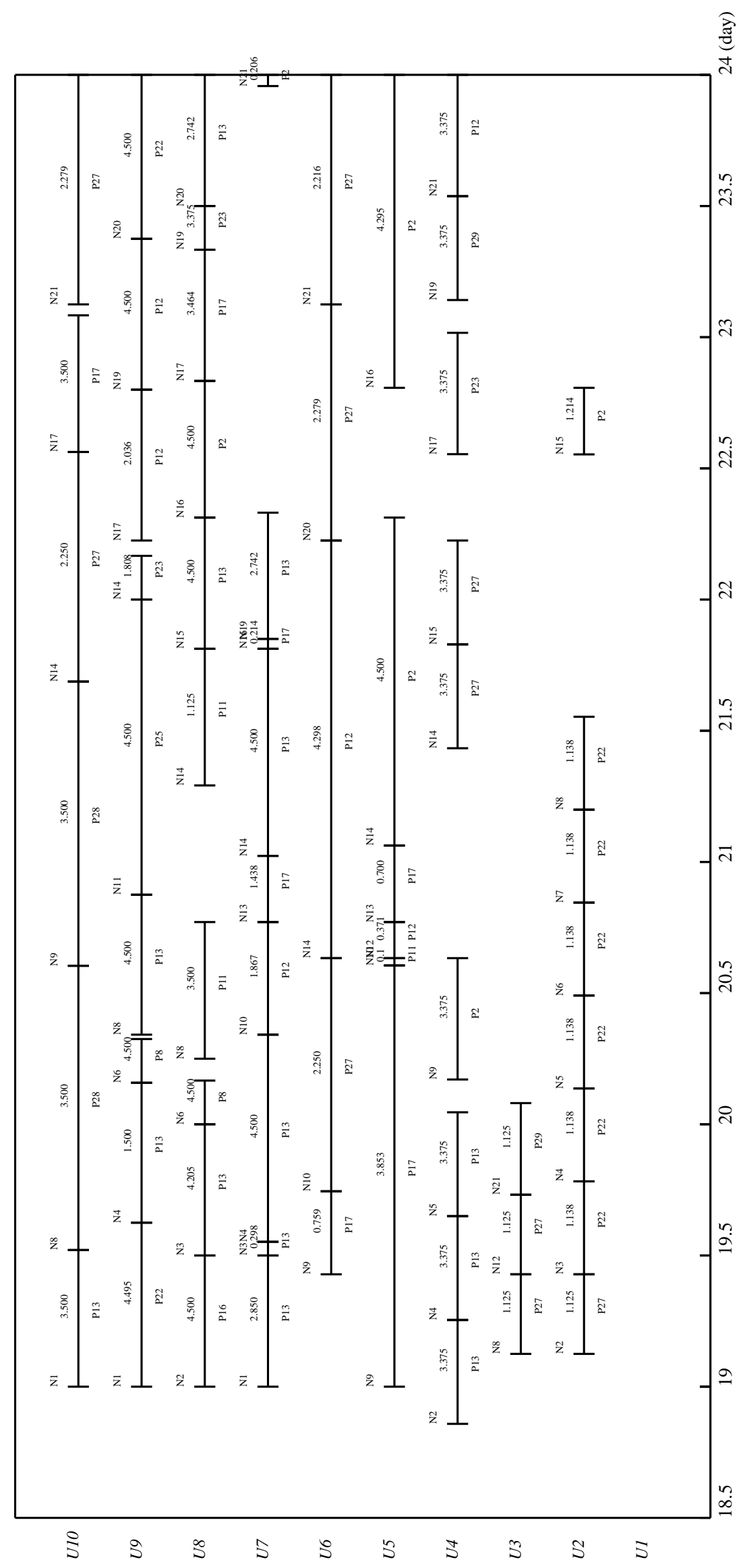

Figure 9: Detailed schedule for Time Horizon 5 (U1-U10: units; P2-P29: products.) 


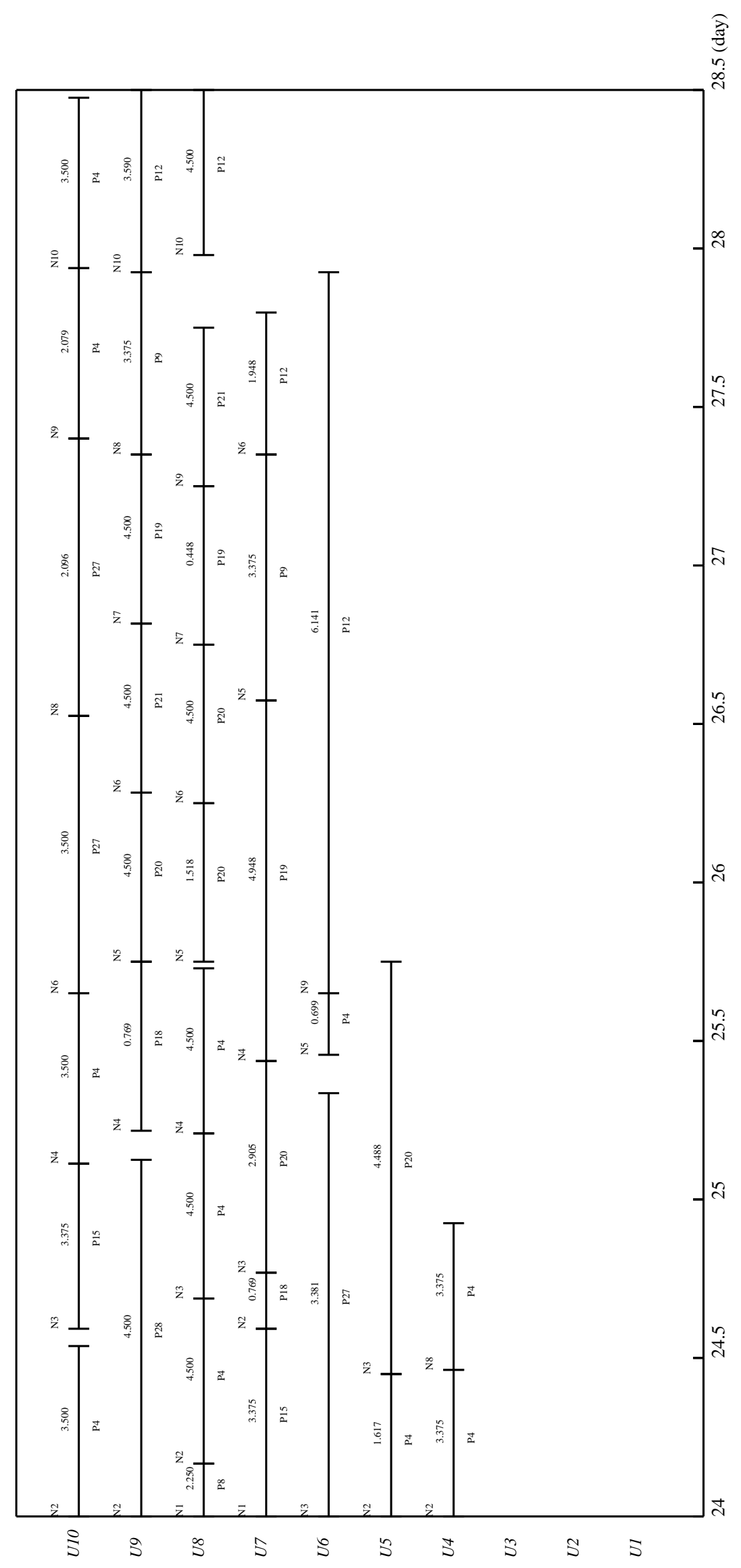

Figure 10: Detailed schedule for Time Horizon 6 (U1-U10: units; P4-P28: products.) 


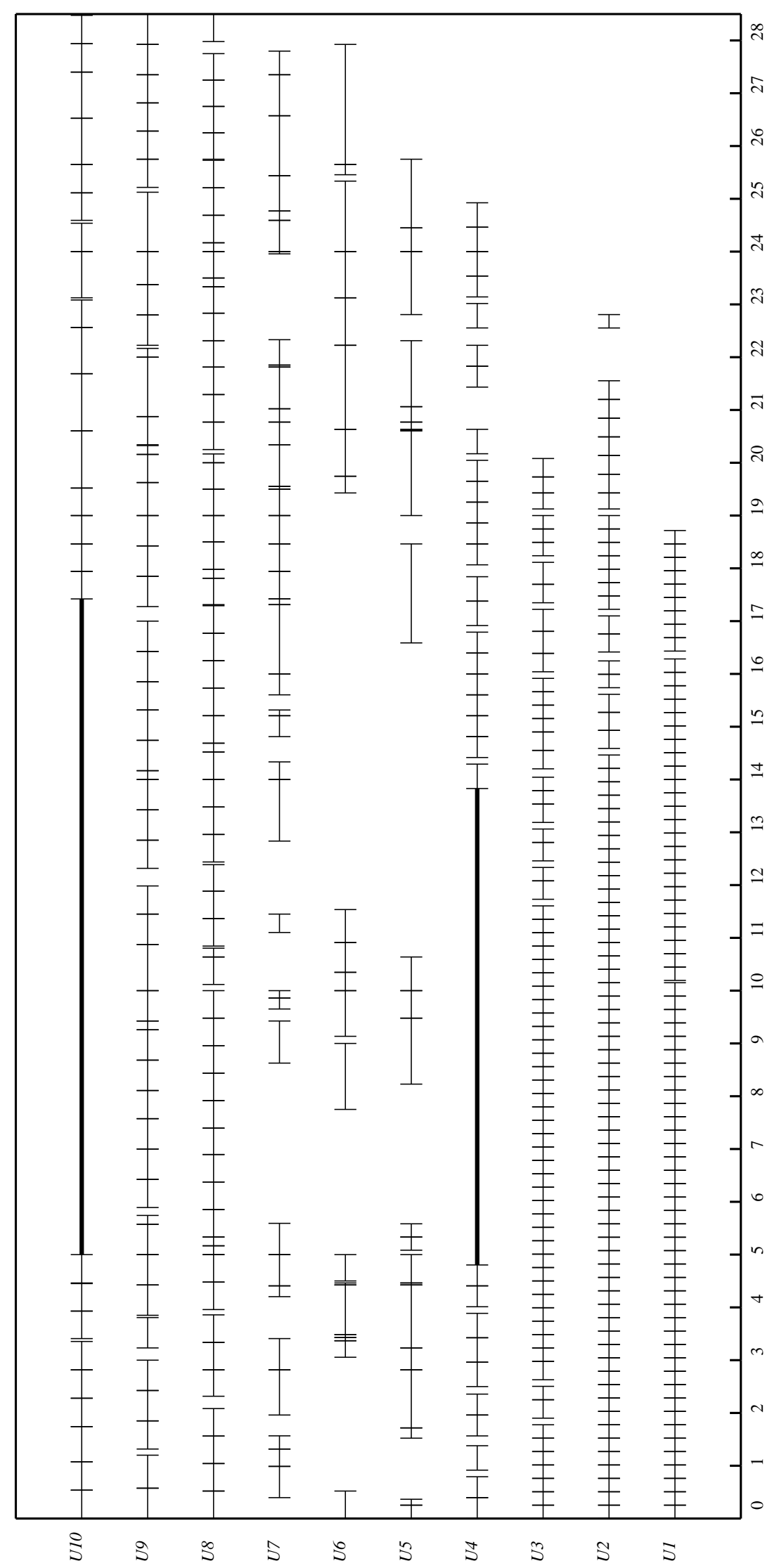

Figure 11: Sketch of production schedule for the whole month (U1-U10: units) 


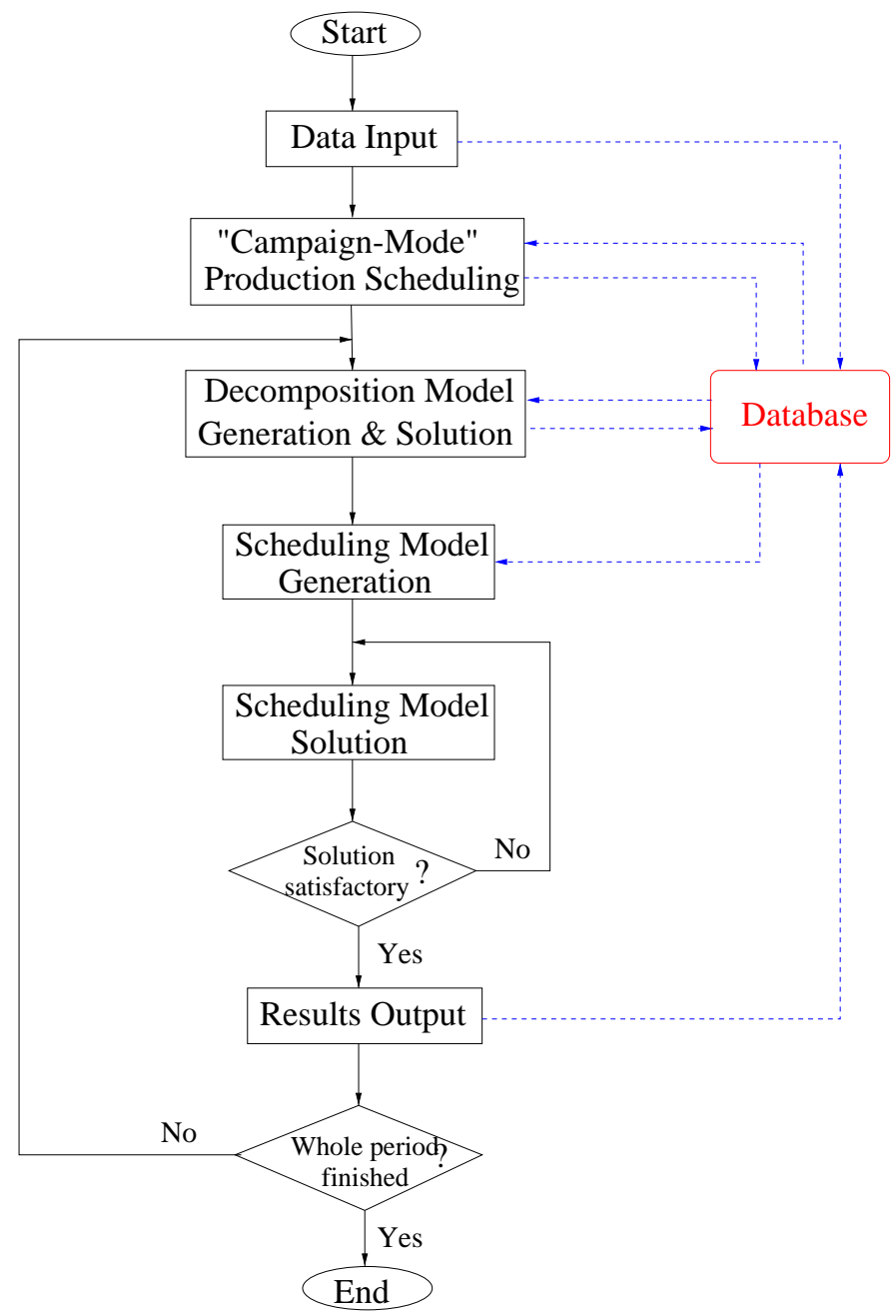

Figure 12: Flowchart of the integrated user interface 


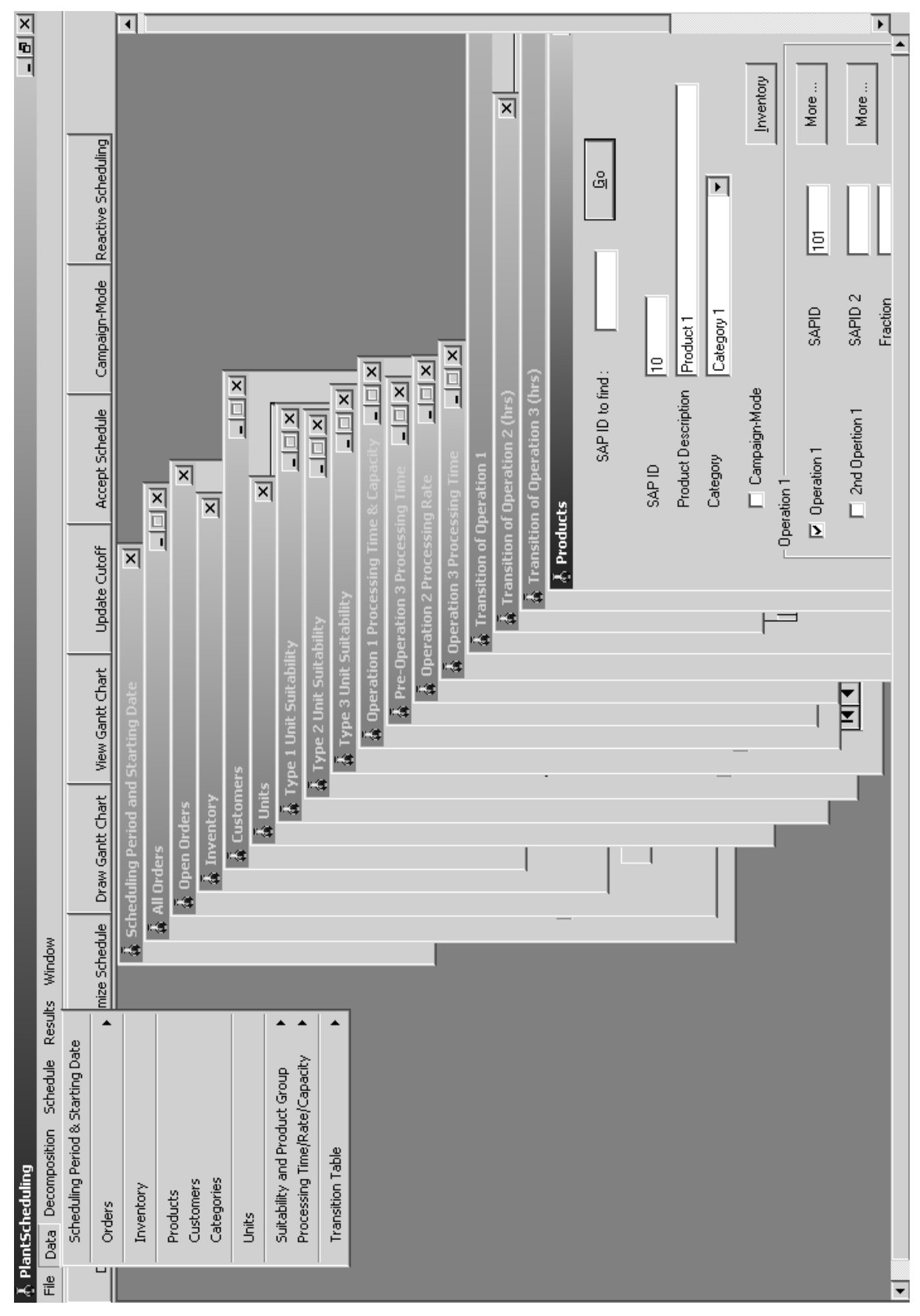

Figure 13: Data forms in the integrated graphical user interface 


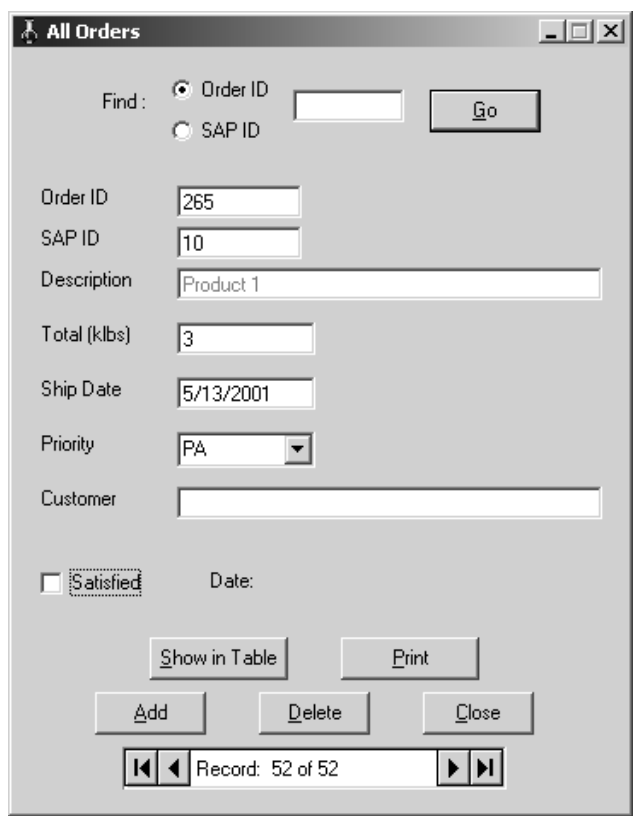

Figure 14: Orders Form in the integrated graphical user interface 


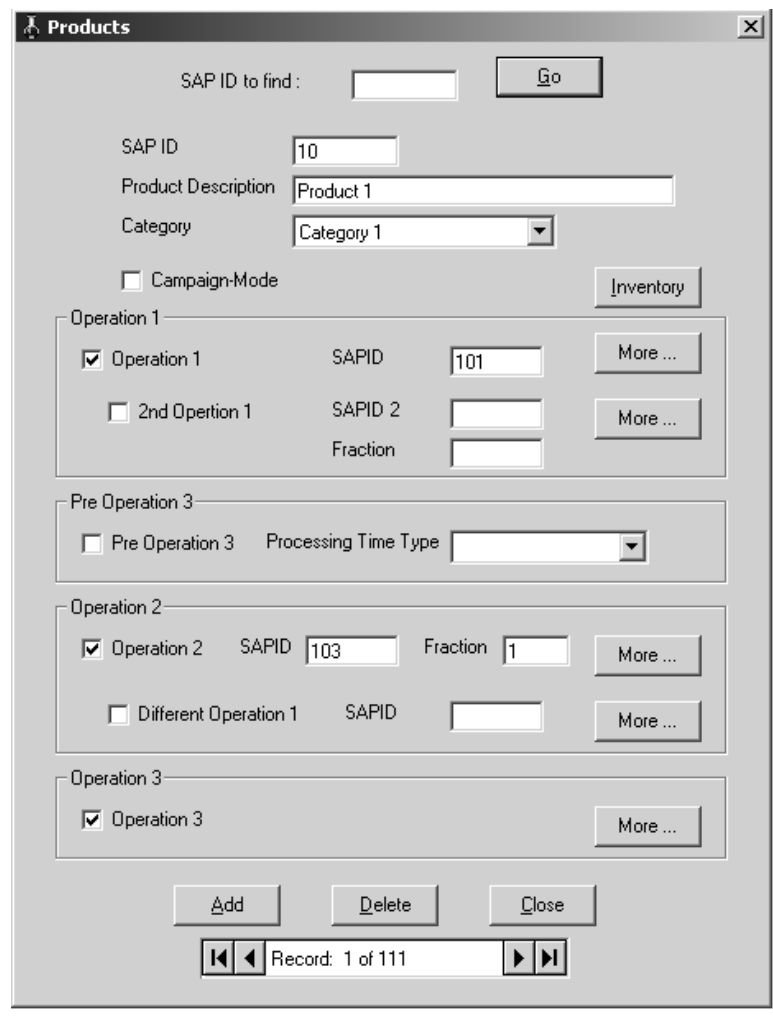

Figure 15: Products Form in the integrated graphical user interface 


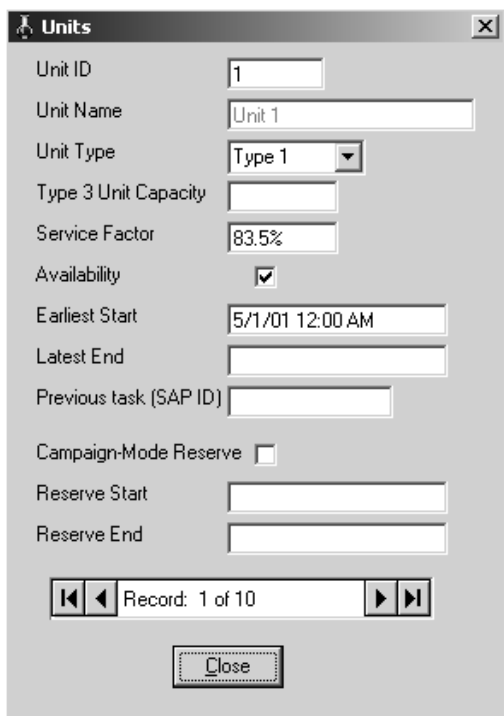

Figure 16: Units Form in the integrated graphical user interface 


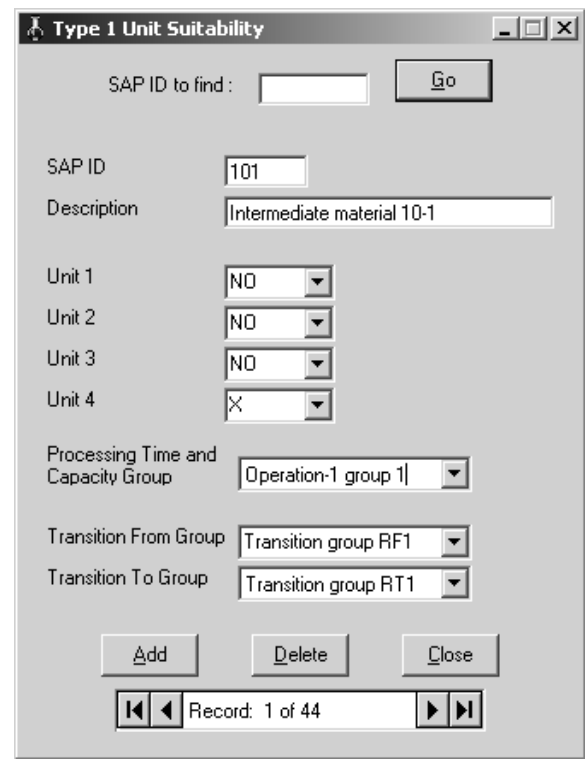

Figure 17: Type 1 Unit Suitability Form in the integrated graphical user interface 


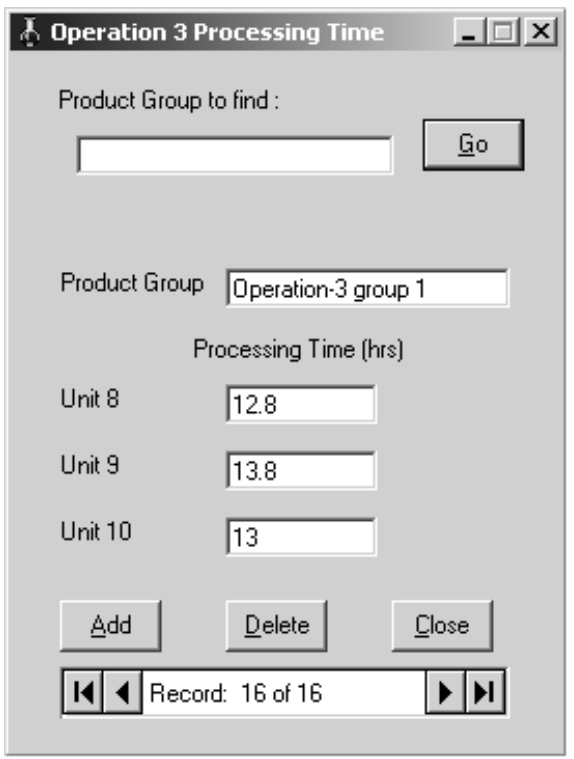

Figure 18: Operation 3 Processing Time Form in the integrated graphical user interface 


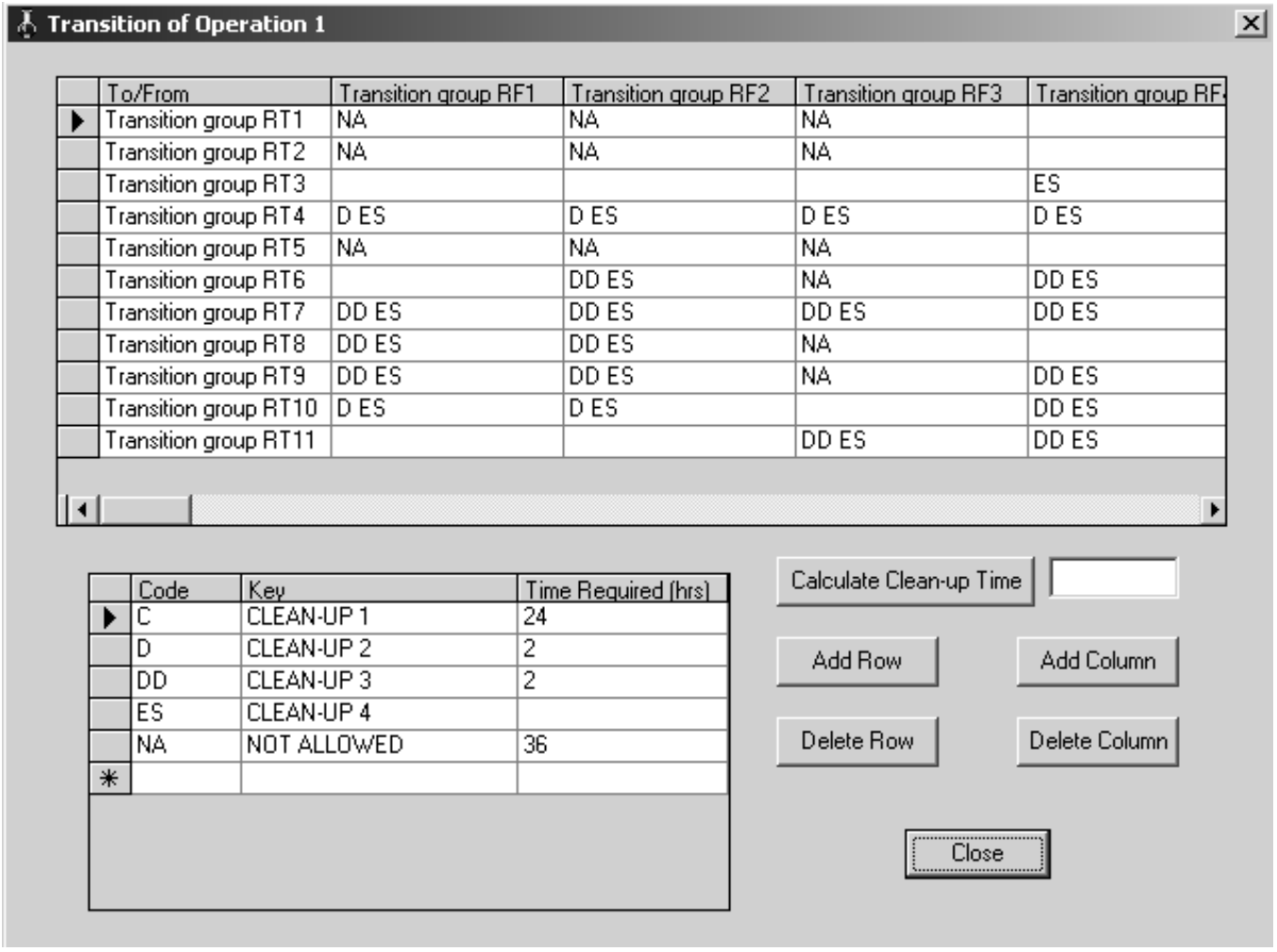

Figure 19: Type 1 Unit Transition Table in the integrated graphical user interface 


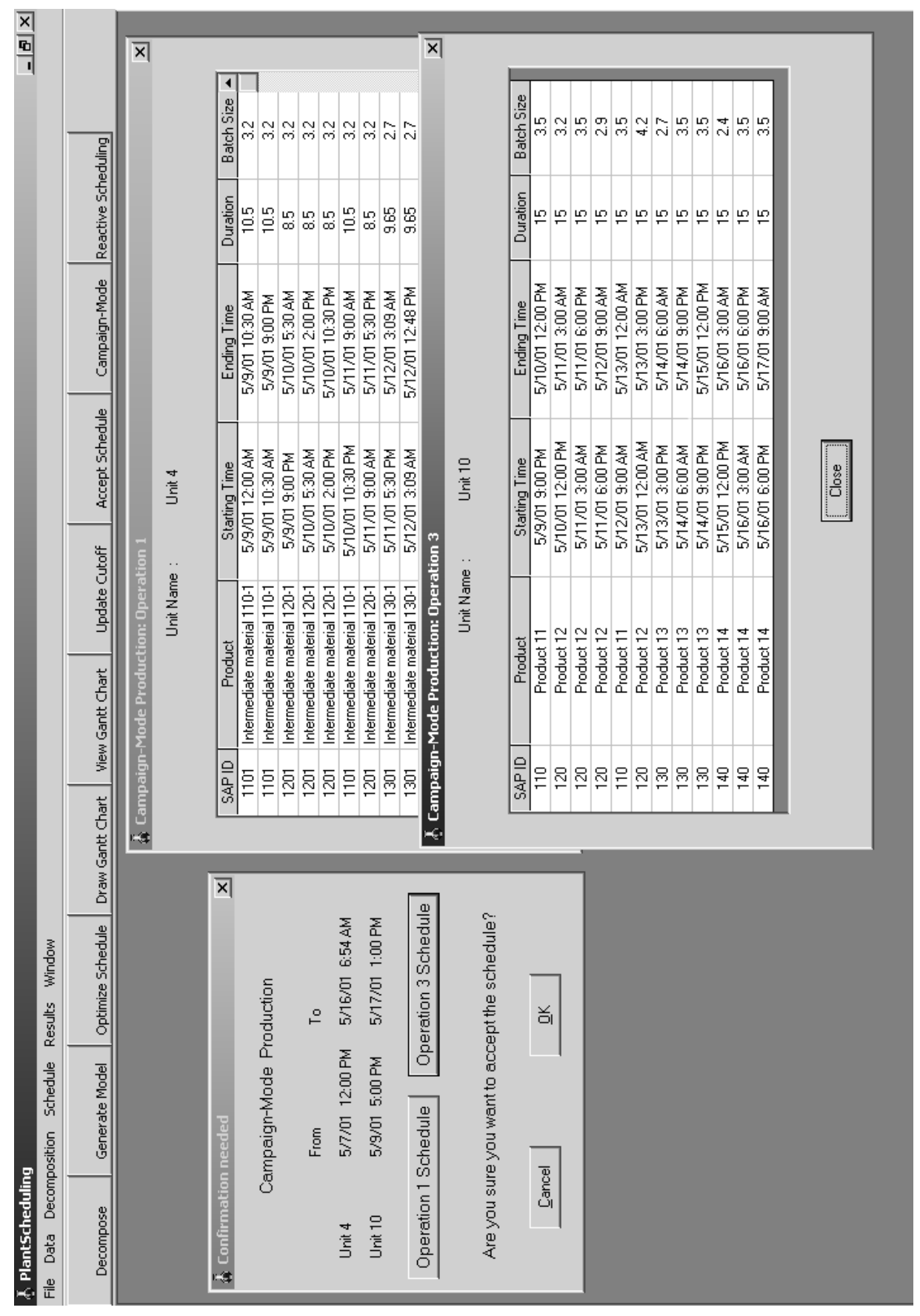

Figure 20: Schedule tables for campaign-mode production obtained in the integrated graphical user interface 


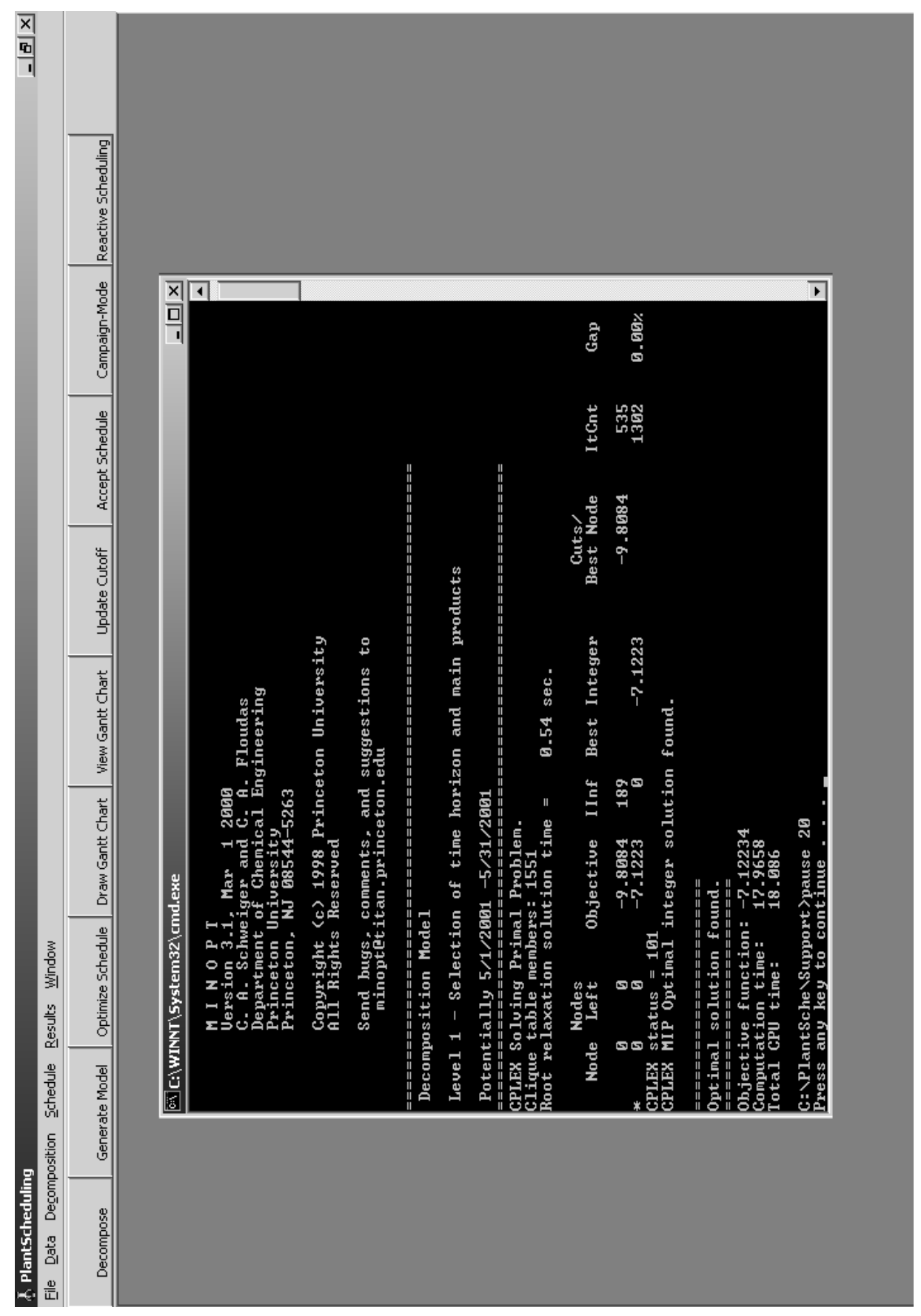

Figure 21: CPLEX solving the decomposition model in the integrated graphical user interface 


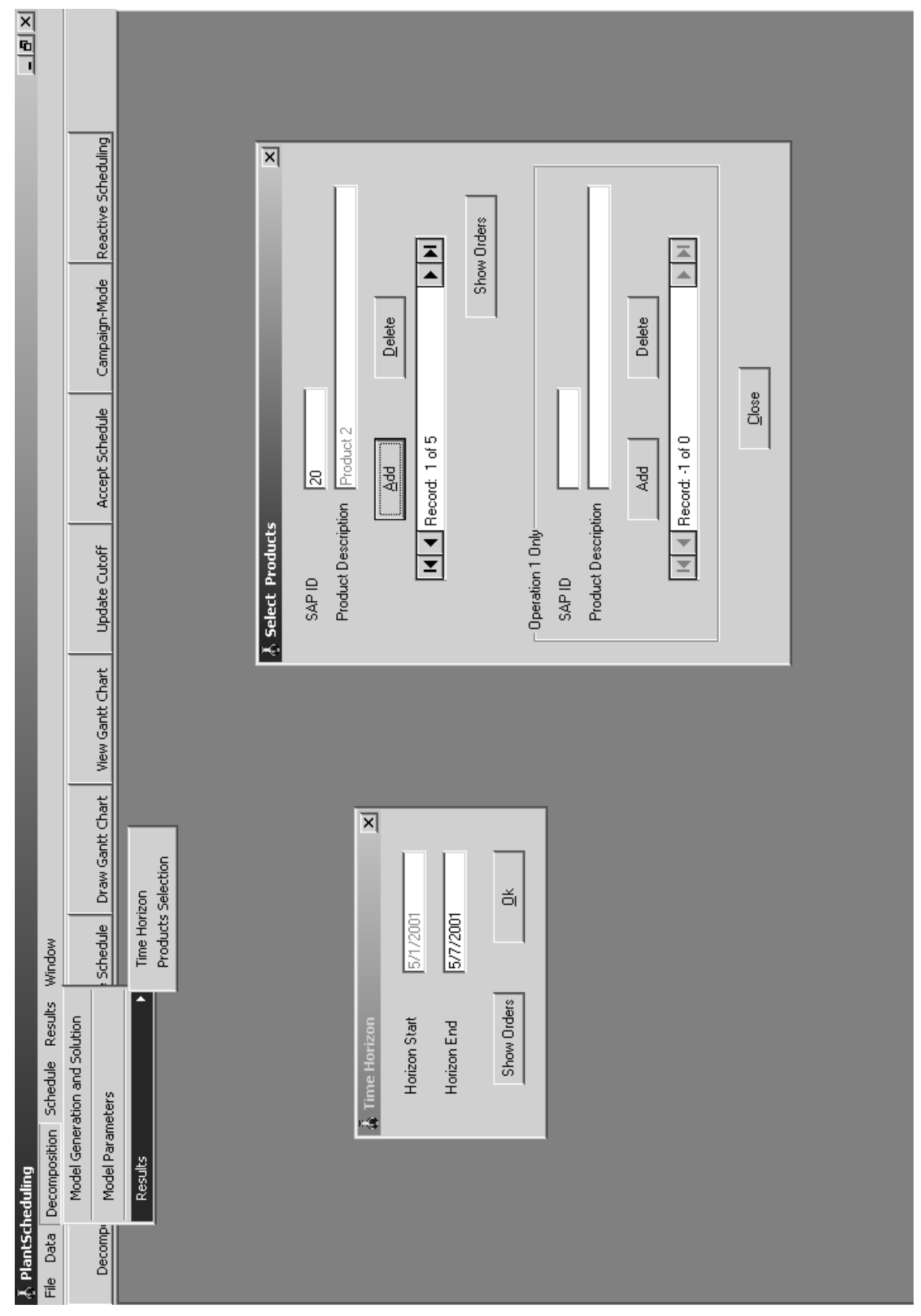

Figure 22: Decomposition results shown in the integrated graphical user interface 


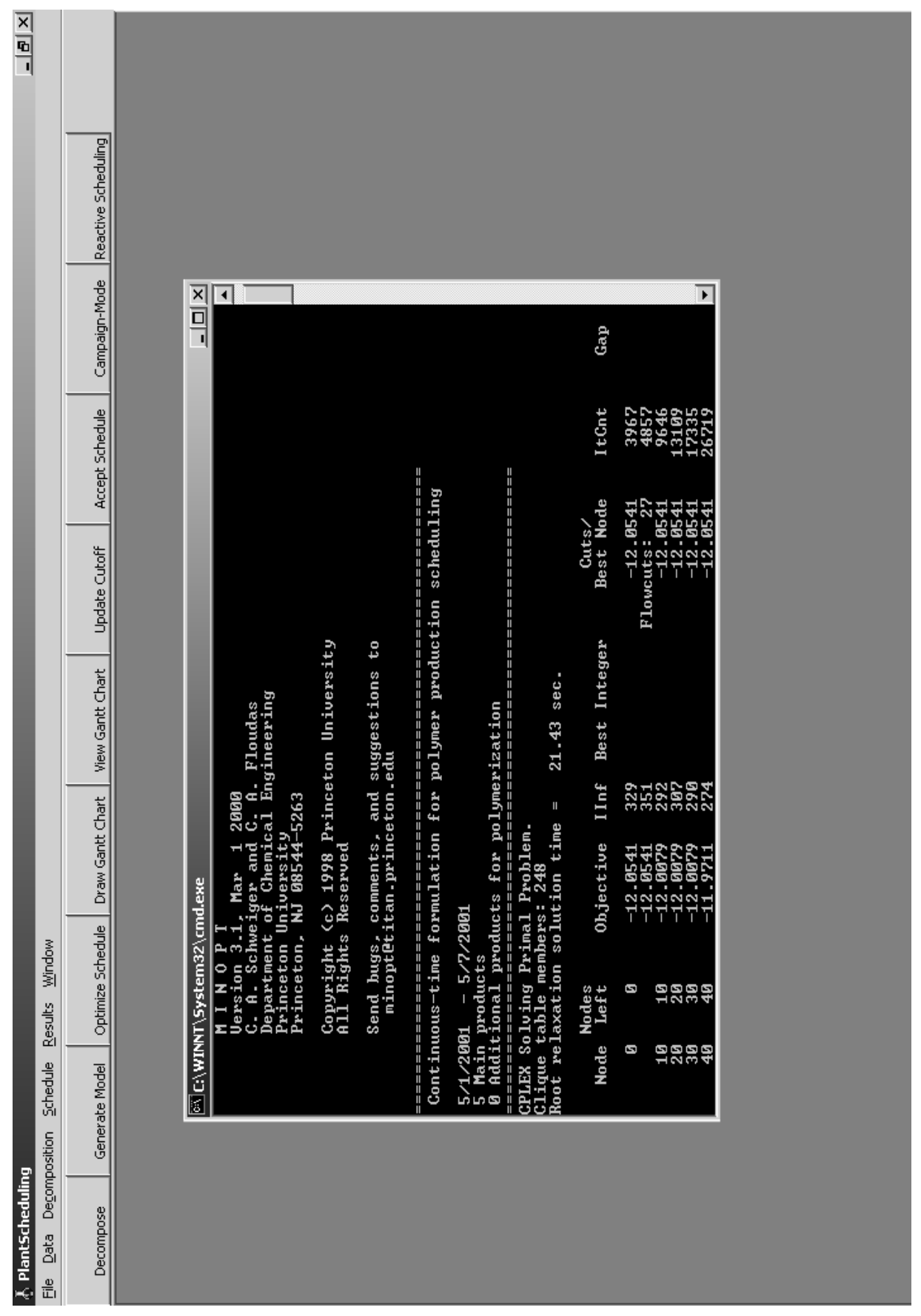

Figure 23: CPLEX solving the short-term scheduling model in the integrated graphical user interface 


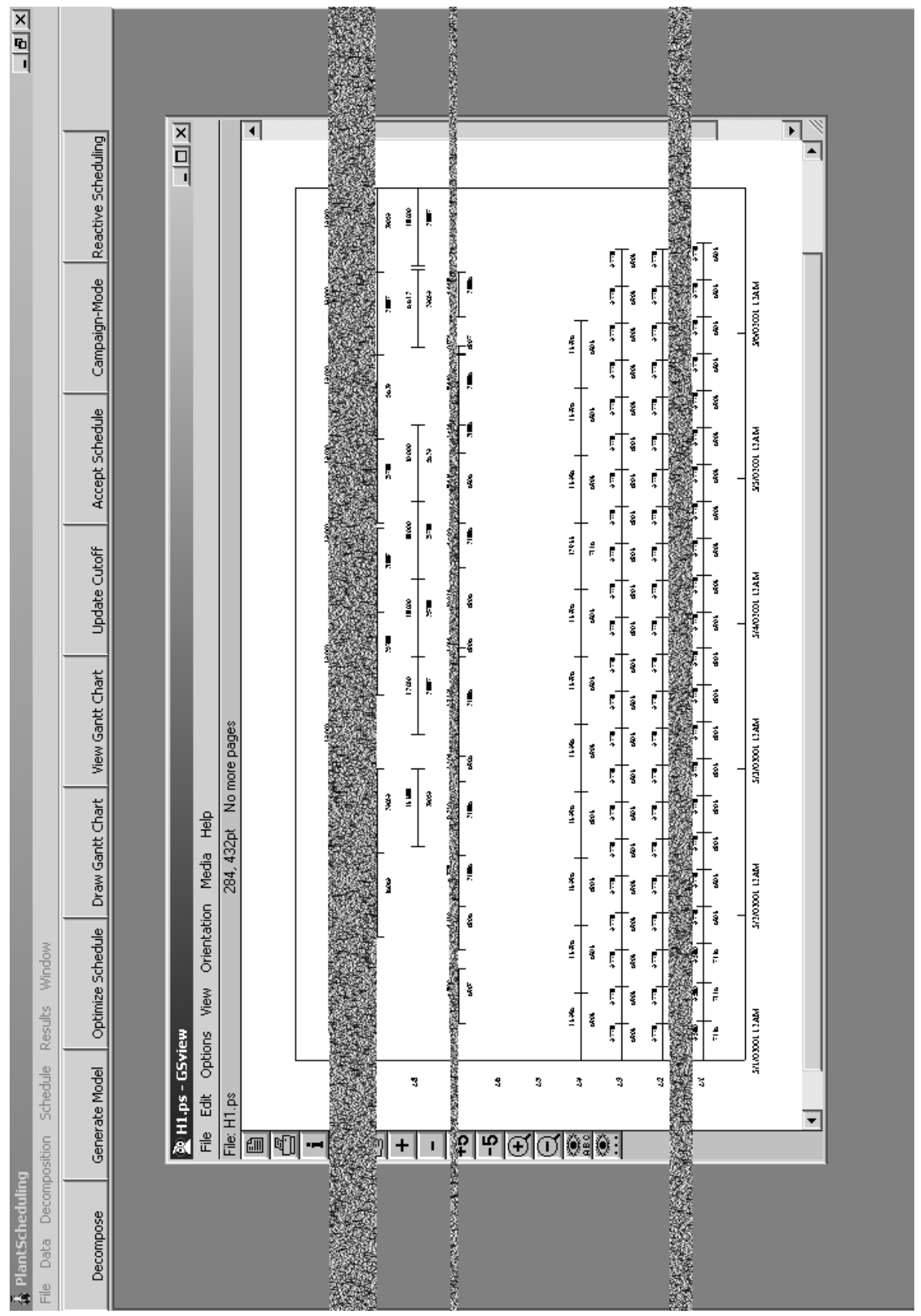

Figure 24: A schedule obtained in the integrated graphical user interface 


\section{List of Tables}

1 Decomposition of the whole scheduling period into successive horizons . . . . . 61

2 Comparisons of demands and production through proposed schedules . . . . . . 62

3 Unit utilization . . . . . . . . . . . . . . . . . . . 63 


\begin{tabular}{c|cccccc}
\hline Time Horizon & 1 & 2 & 3 & 4 & 5 & 6 \\
\hline number of days & 5 & 5 & 4 & 5 & 5 & 4.5 \\
number of main products & 8 & 6 & 10 & 8 & 9 & 11 \\
number of additional products & 0 & 0 & 0 & 7 & 5 & 0 \\
\hline
\end{tabular}

Table 1: Decomposition of the whole scheduling period into successive horizons 


\begin{tabular}{c|cc}
\hline Product & Demand & Production \\
\hline Category 1 & 325.8 & 350.6 \\
\hline Category 2 & 105 & 113.5 \\
\hline Category 3 & 16.5 & 17.0 \\
\hline Category 4 & 54.5 & 61.1 \\
\hline Category 5 & 36.9 & 45.3 \\
\hline Overall & 538.7 & $587.5(+9.1 \%)$ \\
\hline
\end{tabular}

Table 2: Comparisons of demands and production through proposed schedules 


\begin{tabular}{c|cccccccccc}
\hline Unit & U1 & U2 & U3 & U4 & U5 & U6 & U7 & U8 & U9 & U10 \\
\hline Time Used (hrs) & 444.6 & 507.3 & 457.1 & 534.8 & 357.2 & 346.9 & 433.8 & 650.0 & 652.4 & 663.8 \\
$\frac{\text { TimeUsed }}{28.5 \text { days }}(\%)$ & 65.0 & 74.2 & 66.8 & 78.2 & 52.2 & 50.7 & 63.4 & 95.0 & 95.4 & 97.1 \\
\hline
\end{tabular}

Table 3: Unit utilization 